\title{
VARIATIONAL FORMULATION OF A MODEL FREE-BOUNDARY PROBLEM
}

\author{
PATRICIA SAAVEDRA AND L. RIDGWAY SCOTT
}

\begin{abstract}
The purpose of this work is to present an error analysis of the numerical approximation by a finite element method of a free-surface problem. The analysis has been done in an abstract model which has many of the features of a free-surface problem for a viscous liquid. We study in this paper how the numerical approximation of the free boundary affects the approximation of the other variables of the problem and vice versa.
\end{abstract}

We present the numerical analysis of a free-boundary problem that is intended to incorporate many of the difficulties found in a class of models of fluid-flow phenomena with free surfaces. One such phenomenon which motivates the current work is the flow of a liquid constrained only partly by a container, that is, in which a part of the boundary of the domain filled by the liquid is an interface with another liquid of much smaller density, and for which surface tension plays a significant role in determining the shape of the free surface. One model for the behavior of such liquids is based on the assumption that the surface tension between the two liquids is proportional to the curvature of the free surface; the constant of proportionality is a physical property of the two fluids. This model has been studied extensively in recently years, both experimentally (cf. Jean and Pritchard [15] and Pritchard [19]), theoretically (cf. Allain [2], Beale [4], Bemelmans [5], Jean [14], Pukhnachov [20], and Solonnikov [27]), asymptotically (Keller and Miksis [16]) and computationally (cf. Cuvelier [10], Ryskin and Leal [23], and Saito and Scriven [24]). Our purpose here is to establish a framework for the analysis of convergence properties of the computational techniques being used. The only previous work that we are aware of in this direction is by Nitsche [18].

In the first section of the paper, we define our model problem in classical terms. In the second section, we construct a variational formulation for the problem that has two new features. One is that it allows the existence of a solution to be proved with weaker assumptions on the data than has been possible

Received February 26, 1990; revised November 20, 1990.

1980 Mathematics Subject Classification (1985 Revision). Primary 65N30.

Key words and phrases. Free surface, finite element method, variational formulation.

This research was done in part while the authors visited the Institute for Mathematics and its Applications, University of Minnesota. This work was supported in part by the National Science Foundation through award number DMS- 8903548 . 
before. But more importantly, it provides a framework in which a finite element method can be defined and analyzed rigorously. The third section of the paper carries out this analysis. Our variational approach is similar to one used in the code FIDAP [28]. Moreover, our analysis of this class of free-surface problems has much in common with the theoretical studies listed in the previous paragraph, except that the Hölder spaces used there are replaced by appropriate Sobolev spaces. This has the advantage of offering a framework in which finite element spaces that are typically used can be viewed as "conforming" to the theory. Unfortunately, the nature of the problem apparently precludes the use of the traditional Hilbert space theory; we require estimates for a power higher than two of the gradient of the finite element approximation. This is a consequence of the nonlinear relationship between the gradient of the field variable (which corresponds to the velocity variable in a flow problem) and the free surface.

We shall make use of the Lebesgue and Sobolev spaces $L_{p}(\Omega)$ and $W_{p}^{m}(\Omega)$, respectively, $1 \leq p \leq \infty, m \in \mathbb{N}$, defined for a bounded open set, $\Omega$, in $\mathbb{R}^{n}, n=1$ or 2 . These spaces are provided with the usual norm $\|\cdot\|_{L^{p}(\Omega)}$ and $\|\cdot\|_{W_{p}^{m}(\Omega)}$, respectively. $\stackrel{\circ}{W}_{p}^{1}(\Omega)$ is the space of those functions in $W_{p}^{1}(\Omega)$ which vanish on the boundary of $\Omega$ in the generalized sense. The inner product in $L_{2}$ is denoted by $\langle\cdot, \cdot\rangle$.

\section{Problem formulation}

Consider a function $\gamma \in W_{\infty}^{1}(0,1)$ such that $\|\gamma\|_{W_{\infty}^{1}(0,1)}<1$, and related to this function define the following sets:

$$
\Omega_{\gamma}=\{(x, y) \mid 0<x<1,0<y<1+\gamma(x)\}
$$

and

$$
\Gamma_{\gamma}=\{(x, y) \mid 0<x<1, y=1+\gamma(x)\} .
$$

Consider also another function $g \in W_{p}^{1}\left(\Omega^{*}\right)$, where $\Omega^{*}$ is some fixed domain such that $\Omega_{\gamma} \subset \Omega^{*}$ for all $\gamma$ under consideration, and suppose $g(x, y)=0$ for $y \geq 1 / 2$. (In the sequel, we shall choose $p>2$; it will also be seen to be sufficient to define $\Omega^{*}=[0,1] \times[0,2]$.) With these assumptions we define the following

Model free-boundary problem. Find $\gamma$ and $u$ such that

$$
\begin{gathered}
\Delta u=0 \quad \text { in } \Omega_{\gamma,} \\
u=g \quad \text { on } \partial \Omega_{\gamma}, \\
\frac{-s \gamma^{\prime \prime}(x)}{\left[1+\gamma^{\prime}(x)^{2}\right]^{1 / 2}}=\frac{\partial u(x, 1+\gamma(x))}{\partial \vec{n}} \quad \forall x \in[0,1], \\
\gamma(0)=\gamma(1)=0 .
\end{gathered}
$$


The parameter $s>0$ plays the role of the "surface tension" and $\vec{n}$ is the outward normal vector to $\Gamma_{\gamma}$. Note that $u$ is a scalar field and that in the balance of terms on the interface we are using a nonlinear term which is not the curvature of $\gamma$. This is done in order to simplify the variational formulation of the problem. In $\S 4$, we describe the simple modifications necessary to obtain results pertaining to models involving the curvature operator.

In $\S 2$ a weak formulation of this problem is proposed which is more suitable for discretization via variational techniques. It is proved in Corollary 2.1 that the weak formulation admits a unique solution $(\gamma, u) \in W_{\infty}^{1}(0,1) \times W_{p}^{1}\left(\Omega_{\gamma}\right)$ for small values of the norm of $g$, provided that $p \in(2, P)$ for some $P>2$. In $\S 3$ we study the discrete approximation of this problem by a finite element method using piecewise linear functions. In Theorem 3.1 we prove that the discrete problem admits a unique solution, and in Theorem 3.2 and Corollary 3.1 we prove the convergence of this method. One of the conclusions of this work is that it is appropriate to use the same order of piecewise polynomials to approximate $u$ and $\gamma$ in order to obtain the optimal order of convergence.

\section{THE WEAK FORMULATION OF PROBLEM (1.1)}

A preliminary weak formulation of problem (1.1) can be given in the usual way by converting the two elliptic problems for $u$ and $\gamma$, respectively, as follows. First introduce bilinear forms

$$
\begin{aligned}
& a_{\gamma}(u, v):=\int_{\Omega_{\gamma}} \nabla u \cdot \nabla v d x, \\
& b(\gamma, \chi):=s \int_{0}^{1} \gamma^{\prime}(x) \chi^{\prime}(x) d x .
\end{aligned}
$$

Then our preliminary variational formulation is: Find $\gamma \in \stackrel{\circ}{W}_{\infty}^{1}(0,1)$ and $u-g \in \stackrel{\circ}{W}_{p}^{1}\left(\Omega_{\gamma}\right)$ such that

$$
\begin{aligned}
a_{\gamma}(u, v) & =0 \quad \forall v \in \stackrel{\circ}{W}_{q}^{1}\left(\Omega_{\gamma}\right), \\
b(\gamma, \chi) & =\int_{0}^{1} \frac{\partial u}{\partial \vec{n}}(x, 1+\gamma(x)) \chi(x)\left[1+\gamma^{\prime}(x)^{2}\right]^{1 / 2} d x \quad \forall \chi \in \stackrel{\circ}{W}_{1}^{1}(0,1) .
\end{aligned}
$$

The shortcoming of this variational formulation is its reference to a quantity, $\partial u / \partial \vec{n}$, that is undefined for arbitrary $u$ in the space in which we seek the solution, $u$. We now address this issue. (Our reasons for choosing non-Hilbert spaces in the variational formulation will be clarified subsequently.)

If $u$ is a sufficiently regular solution of problem (1.1) then Green's formula implies

$$
\begin{array}{r}
\int_{0}^{1} \frac{\partial u}{\partial \vec{n}}(x, 1+\gamma(x)) \chi(x)\left[1+\gamma^{\prime}(x)^{2}\right]^{1 / 2} d x \\
=\int_{\Gamma_{y}} \frac{\partial u}{\partial \vec{n}} v d s=\int_{\Omega_{\gamma}} \nabla u \cdot \nabla v d x,
\end{array}
$$

provided that $v(x, 1+\gamma(x))=\chi(x)$ and $v=0$ on $\partial \Omega_{\gamma} \backslash \Gamma_{\gamma}$. 
Lemma 2.0. For all $\chi \in W_{1}^{1}(0,1)$ it is possible to construct an extension $E_{\gamma} \chi \in$ $W_{q}^{1}\left(\Omega_{\gamma}\right)$ whenever $q<2$, such that $\left.E_{\gamma} \chi\right|_{\Gamma_{\gamma}}=\chi$ and $\left.E_{\gamma} \chi\right|_{\left.\partial \Omega_{\gamma}\right\rangle \Gamma_{\gamma}}=0$.

Proof. First, by one of Sobolev's imbedding theorems, $\chi \in W_{1}^{1}(0,1)$ implies $\chi \in W_{q}^{1-1 / q}(0,1)$ for $q<2$. If $\chi \in W_{q}^{1-1 / q}(0,1)$ for $q<2$, we can extend $\chi$ by zero to $\partial \Omega_{0}$, where $\Omega_{0}=[0,1] \times[0,1]$, and assure that this extension $\tilde{\chi} \in W_{q}^{1-1 / q}\left(\partial \Omega_{0}\right)$. By the surjectivity property of the trace operator, there is a $\hat{v} \in W_{q}^{1}\left(\Omega_{0}\right)$ such that $\left.\hat{v}\right|_{\partial \Omega_{0}}=\tilde{\chi}$. Let $\hat{E} \chi$ denote this extension, $\hat{v}$. Since the embedding and trace are continuous operators, there exist $C, C_{E}<\infty$, independent of $\chi$, such that

$$
\|\widehat{E} \chi\|_{W_{q}^{1}\left(\Omega_{0}\right)} \leq C\|\chi\|_{W_{q}^{1-1 / q}\left(\partial \Omega_{0}\right)} \leq C_{E}\|\chi\|_{W_{1}^{1}(0,1)} .
$$

(For details, see Grisvard [13] and Arnold, Scott and Vogelius [3].)

Now we transform the domain $\Omega_{0}$ to $\Omega_{\gamma}$ by the following change of variables:

$$
(\xi, \eta) \in \Omega_{0} \longrightarrow(x, y) \in \Omega_{\gamma}, \quad x=\xi \text { and } y=(1+\gamma(\xi)) \eta .
$$

This induces a mapping on functions, $v$, on $\Omega_{\gamma}$ to functions, $\hat{v}$, on $\Omega_{0}$ via

$$
\hat{v}(\xi, \eta)=v(\xi,(1+\gamma(\xi)) \eta) .
$$

If $\|\gamma\|_{W_{\infty}^{1}(0,1)}<1$, then the mappings (2.3) are invertible, and for any $\hat{v} \in$ $W_{q}^{1}\left(\Omega_{0}\right)$ there is a $v \in W_{q}^{1}\left(\Omega_{\gamma}\right)$ such that

$$
v(x, y)=\hat{v}(x, y /(1+\gamma(x))) .
$$

Moreover, we have

$$
\begin{aligned}
& \|v\|_{W_{q}^{1}\left(\Omega_{\gamma}\right)} \leq \mu\left(\|\gamma\|_{W_{\infty}^{1}(0,1)}\right)\|\hat{v}\|_{W_{q}^{1}\left(\Omega_{0}\right)}, \\
& \|\hat{v}\|_{W_{q}^{1}\left(\Omega_{0}\right)} \leq \mu\left(\|\gamma\|_{W_{\infty}^{1}(0,1)}\right)\|v\|_{W_{q}^{1}\left(\Omega_{\gamma}\right)},
\end{aligned}
$$

where $\mu(t)$ is a continuous function for $0 \leq t<1$ and $q$ may be any real number in the range $1 \leq q \leq \infty$. Now we can identify $\chi$ with its extension $E_{\gamma} \chi$ in $\Omega_{\gamma}$ by $\widehat{E_{\gamma} \chi}:=\widehat{E} \chi$, i.e.,

$$
E_{\gamma} \chi(x, y)=\widehat{E} \chi(x, y /(1+\gamma(x))),
$$

where $\widehat{E} \chi$ was defined above extending $\chi$ to $\Omega_{0}$. From (2.6) and (2.2) we conclude

$$
\begin{aligned}
\left\|E_{\gamma} \chi\right\|_{W_{q}^{1}\left(\Omega_{\gamma}\right)} & \leq \mu\left(\|\gamma\|_{W_{\infty}^{1}(0,1)}\right)\|\widehat{E} \chi\|_{W_{q}^{1}\left(\Omega_{0}\right)} \\
& \leq C_{E} \mu\left(\|\gamma\|_{W_{\infty}^{1}(0,1)}\right)\|\chi\|_{W_{1}^{1}(0,1)}
\end{aligned}
$$

Remark. No extension is possible for $q \geq 2$, even for $\chi \in \stackrel{\circ}{W}_{1}^{1}(0,1)$. To prove this, pick a sequence $\phi_{j}$ such that $\phi_{j}=1$ in $\left[\frac{1}{4}, \frac{3}{4}\right], \phi_{j}=0$ outside 
$\left[\frac{1}{4}-1 / j, \frac{3}{4}+1 / j\right]$ and $\phi_{j}$ is linear in $\left[\frac{1}{4}-1 / j, \frac{1}{4}\right]$ and $\left[\frac{3}{4}, \frac{3}{4}+1 / j\right]$. This sequence is bounded in $\stackrel{\circ}{W}_{1}^{1}(0,1)$. However, it is not bounded in $H^{\frac{1}{2}}(0,1)$. This can be seen either by direct computation using Fourier transforms or by observing that the $\phi_{j}$ 's tend to the characteristic function of $\left[\frac{1}{4}, \frac{3}{4}\right]$ and that the latter is not in $H^{\frac{1}{2}}(0,1)$. Since the $\phi_{i}$ 's do not remain bounded in $H^{\frac{1}{2}}(0,1)$, they cannot be extended boundedly to $H^{1}\left(\Omega_{0}\right)$ [6].

Now equation (2.1) can be inserted in our preliminary weak formulation to yield our final weak formulation of problem (1.1) (for $p>2$ and $1 / p+1 / q=$ $1)$.

Variational free-boundary problem. Find $\gamma \in \stackrel{\circ}{W}_{\infty}^{1}(0,1)$ and $u \in g \oplus \stackrel{\circ}{W}_{p}^{1}\left(\Omega_{\gamma}\right)$ such that

$$
\begin{array}{ll}
a_{\gamma}(u, v)=0 & \forall v \in \stackrel{\circ}{W}_{q}^{1}\left(\Omega_{\gamma}\right), \\
b(\gamma, \chi)=a_{\gamma}\left(u, E_{\gamma} \chi\right) & \forall \chi \in \stackrel{\circ}{W}_{1}^{1}(0,1) .
\end{array}
$$

Remark. The problem (2.9) does not depend on the extension $E_{\gamma}$ because

$$
a_{\gamma}\left(u, E_{\gamma}^{1} \chi-E_{\gamma}^{2} \chi\right)=0 \quad \forall \chi \in \stackrel{\circ}{W}_{1}^{1}(0,1)
$$

for two such extensions, since $E_{\gamma}^{1} \chi-E_{\gamma}^{2} \chi \in \stackrel{\circ}{W}_{q}^{1}\left(\Omega_{\gamma}\right)$. Moreover, $a_{\gamma}\left(u, E_{\gamma} \chi\right)$ can be evaluated via $(2.1)$, that is

$$
\begin{aligned}
a_{\gamma}\left(u, E_{\gamma} \chi\right) & =\int_{\Omega_{\gamma}} \nabla u \cdot \nabla E_{\gamma} \chi d x=\int_{\Gamma_{\gamma}} \frac{\partial u}{\partial \vec{n}} E_{\gamma} \chi d s \\
& =\int_{0}^{1} \frac{\partial u}{\partial \vec{n}}(x, 1+\gamma(x)) \chi(x)\left[1+\gamma^{\prime}(x)^{2}\right]^{1 / 2} d x,
\end{aligned}
$$

provided $u$ and $\chi$ are regular enough.

To establish the existence of a solution $(\gamma, u)$ of $(2.9)$, we shall use a contraction argument. Before presenting this result, some a priori estimates will be proved. First define the following seminorms in $W_{p}^{1}(0,1)$ :

$$
|\gamma|_{W_{p}^{\prime}(0,1)}=\left\|\gamma^{\prime}\right\|_{L^{p}(0,1)} \quad \forall \gamma \in W_{p}^{1}(0,1), \quad 1 \leq p \leq \infty .
$$

The following is a simple consequence of Rellich's theorem; cf. Brezis [7].

Proposition 2.1. $|\cdot|_{W_{p}^{1}(0,1)}$ is a norm in $\stackrel{\circ}{W}_{p}^{1}(0,1)$ equivalent to $\|\cdot\|_{W_{p}^{1}(0,1)}$ for $1 \leq p \leq \infty$.

Proposition 2.2. The bilinear form, $b$, is continuous from $W_{\infty}^{1}(0,1) \times W_{1}^{1}(0,1)$ $\rightarrow \mathbb{R}$. Furthermore, there exists a constant $\beta<\infty$ such that for all $\gamma \in$ $\stackrel{\circ}{W}_{\infty}^{1}(0,1)$

$$
\|\gamma\|_{W_{\infty}^{1}(0,1)} \leq \beta \sup _{\substack{\infty \\ 0 \neq \chi \in W_{1}^{1}(0,1)}} \frac{b(\gamma, \chi)}{\|\chi\|_{W_{1}^{1}(0,1)}} .
$$


Proof. The continuity of $b$ is a consequence of Hölder's inequality. On the other hand, Proposition 2.1 implies that for all $\gamma \in \stackrel{\circ}{W}_{\infty}^{1}(0,1)$

$$
\begin{aligned}
\|\gamma\|_{W_{\infty}^{1}(0,1)} & \leq C\left\|\gamma^{\prime}\right\|_{L^{\infty}(0,1)}=C \sup _{\substack{f \in L^{\prime}(0,1) \\
f \neq 0}} \frac{\left\langle\gamma^{\prime}, f\right\rangle}{\|f\|_{L^{1}(0,1)}} \\
& =-C \sup _{\substack{f \in L^{\prime}(0,1) \\
f \neq 0}} \frac{\left\langle\gamma^{\prime}, f-\bar{f}\right\rangle}{\|f\|_{L^{1}(0,1)}} \leq 2 C \sup _{\substack{f \in L^{\prime}(0,1) \\
f-\bar{f} \neq 0}} \frac{\left\langle\gamma^{\prime}, f-\bar{f}\right\rangle}{\|f-\bar{f}\|_{L^{1}(0,1)}},
\end{aligned}
$$

where $\bar{f}:=\int_{0}^{1} f(x) d x$ and we have used the simple inequality $\|f-\bar{f}\|_{L^{1}(0,1)} \leq$ $2\|f\|_{L^{1}(0,1)}$. For any $f \in L^{1}(0,1)$, there is a $\chi \in \stackrel{\circ}{W}_{1}^{1}(0,1)$ such that $\chi^{\prime}(x)=$ $f(x)-\bar{f}$. Using again the fact that $|\cdot|_{W_{1}^{1}(0,1)}$ and $\|\cdot\|_{W_{1}^{1}(0,1)}$ are equivalent norms in $\stackrel{\circ}{W}_{1}^{1}(0,1)$, we have

$$
\begin{aligned}
\|\gamma\|_{W_{\infty}^{1}(0,1)} & \leq C \sup _{\substack{\circ \\
0 \neq \chi \in W_{1}^{1}(0,1)}} \frac{\int_{0}^{1} \gamma^{\prime}(x) \chi^{\prime}(x) d x}{\left\|\chi^{\prime}\right\|_{L^{1}(0,1)}} \\
& \leq \frac{C}{S} \sup _{\substack{\stackrel{\circ}{W}_{1}^{\prime}(0,1) \\
0 \neq \chi \in \|_{1}}} \frac{b(\gamma, \chi)}{\|\chi\|_{W_{1}^{1}(0,1)}},
\end{aligned}
$$

which proves inequality (2.10) with $\beta=\frac{C}{s}$.

Remark. In the previous proof and in subsequent estimates, $C$ denotes a generic constant that may increase from line to line but is dependent only on the stated quantities.

Proposition 2.3. Suppose $\|\gamma\|_{W_{\infty}^{1}(0,1)}<1 / 2$. Then $a_{\gamma}$ is a continuous bilinear form from $W_{p}^{1}\left(\Omega_{\gamma}\right) \times W_{q}^{1}\left(\Omega_{\gamma}\right) \rightarrow \mathbb{R}$, where $1 / p+1 / q=1$. Moreover, there are constants $\alpha<\infty$ and $Q<2<P$ such that for all $u \in \stackrel{\circ}{W}_{p}^{1}\left(\Omega_{\gamma}\right)$

$$
\|u\|_{W_{p}^{1}\left(\Omega_{\gamma}\right)} \leq \alpha \sup _{\substack{0 \neq v \in \dot{W}_{q}^{1}\left(\Omega_{\gamma}\right)\\}} \frac{a_{\gamma}(u, v)}{\|v\|_{W_{q}^{1}\left(\Omega_{\gamma}\right)}},
$$

whenever $Q \leq p \leq P$.

Proof. The first part of the theorem is easily proved using Hölder's inequality. The second part will be proved using a result of Meyers [17] concerning secondorder elliptic divergence equations with bounded, measurable coefficients. To use this result, we transform the domain $\Omega_{\gamma}$ to $\Omega_{0}$, using the change of variables (2.3). Thus, for all $u \in W_{p}^{1}\left(\Omega_{\gamma}\right)$ and $v \in W_{q}^{1}\left(\Omega_{\gamma}\right)$, we have $\hat{u} \in W_{p}^{1}\left(\Omega_{0}\right)$ and $\hat{v} \in W_{q}^{1}\left(\Omega_{0}\right)$ such that

$$
a_{\gamma}(u, v)=\int_{\Omega_{0}} \sum_{i, j=1}^{2} A_{i j}(\xi, \eta ; \gamma) \partial_{i} \hat{u} \partial_{j} \hat{v} d \xi d \eta=: \hat{a}(\hat{u}, \hat{v} ; \gamma),
$$


where $\partial_{1} u=\partial u / \partial \xi$ and $\partial_{2} u=\partial u / \partial \eta$, and

$$
\begin{gathered}
A_{11}(\xi, \eta ; \gamma)=1+\gamma(\xi), \quad A_{22}(\xi, \eta ; \gamma)=\frac{1+\gamma^{\prime}(\xi)^{2} \eta^{2}}{1+\gamma(\xi)}, \\
A_{12}(\xi, \eta ; \gamma)=A_{21}(\xi, \eta ; \gamma)=-\gamma^{\prime}(\xi) \eta .
\end{gathered}
$$

Note that $A=\left(D D^{T}\right)^{-1}|\operatorname{det} D|$, where $D$ is the Jacobian of the mapping (2.3). For all $\gamma \in W_{\infty}^{1}(0,1)$ such that $\|\gamma\|_{W_{\infty}^{1}(0,1)}<1 / 2, A_{i j}$ is well defined a.e. in $\Omega_{0}$ for $1 \leq i, j \leq 2$, and the following holds: $A$ is a real, symmetric matrix such that

(2.14) $\exists \Lambda>0$ such that $A_{i j}(\xi, \eta) z_{j} z_{i} \geq \Lambda z_{i}^{2} \quad \forall z \in \mathbb{R}^{2}$, a.a. $(\xi, \eta) \in \Omega_{0}$,

$$
\exists M<\infty \text { such that } \max _{1 \leq i, j \leq 2}\left\|A_{i j}\right\|_{L^{\infty}\left(\Omega_{0}\right)} \leq M .
$$

Indeed, (2.15) is a consequence of the assumption $\|\gamma\|_{W_{\infty}^{1}(0,1)}<1 / 2$, and (2.15) implies (2.14) as follows. The eigenvalues of $\left(A_{i j}(\xi, \eta)\right)$ satisfy $\lambda_{\min }(\xi, \eta) \leq$ $\lambda_{\text {max }}(\xi, \eta) \leq 2 M$. Since $\operatorname{det} A=1$, we have $\lambda_{\min }=\lambda_{\max }^{-1} \geq 1 / 2 M=: \Lambda$, which implies (2.14).

From Meyers [17] it follows that there are real numbers $Q<2<P$ and a finite constant $\alpha$ such that

$$
\|\hat{u}\|_{W_{p}^{\prime}\left(\Omega_{0}\right)} \leq \alpha \sup _{\substack{0 \neq \hat{v} \in W_{q}^{\prime}\left(\Omega_{0}\right)\\}} \frac{\hat{a}(\hat{u}, \hat{v} ; \gamma)}{\|\hat{v}\|_{W_{q}^{\prime}\left(\Omega_{0}\right)}}
$$

whenever $Q<p<P$. In view of (2.12), the result follows from (2.16) and the equivalence of norms $(2.6)$.

Existence results. For $\varepsilon>0$, consider the following set

$$
V_{\varepsilon}=\left\{(\gamma, u) \in \stackrel{\circ}{W}_{\infty}^{1}(0,1) \times W_{p}^{1}\left(\Omega_{\gamma}\right):\|\gamma\|_{W_{\infty}^{1}(0,1)}<\frac{1}{2},\|u\|_{W_{p}^{1}\left(\Omega_{y}\right)}<\varepsilon\right\},
$$

and let $T$ be the following mapping: given $(\gamma, u) \in V_{\varepsilon}$, let

$$
T(\gamma, u)=\left(T_{1}(\gamma, u), T_{2}(\gamma, u)\right)=(\tilde{\gamma}, \tilde{u}),
$$

where $(\tilde{\gamma}, \tilde{u})$ is determined as follows. First, $\tilde{\gamma} \in \stackrel{\circ}{W}_{\infty}^{1}(0,1)$ is found satisfying

$$
b(\tilde{\gamma}, \chi)=a_{\gamma}\left(u, E_{\gamma}(\chi)\right) \quad \forall \chi \in \stackrel{\circ}{W}_{1}^{1}(0,1) .
$$

Then $\tilde{u}-g \in W_{p}^{1}\left(\Omega_{\tilde{\gamma}}\right)$ solves

$$
a_{\tilde{\gamma}}(\tilde{u}, v)=0 \quad \forall v \in \stackrel{\circ}{W}_{q}^{1}\left(\Omega_{\tilde{\gamma}}\right) .
$$

Lemma 2.1. There exist two positive real numbers $\delta$ and $\varepsilon$ and a real number $P>2$ such that, if $\|g\|_{W_{p}^{1}\left(\Omega^{*}\right)}<\delta$, then $T$ is a mapping from $V_{\varepsilon} \rightarrow V_{\varepsilon}$ whenever $p \in(2, P)$.

Proof. Let $(\gamma, u) \in V_{\varepsilon}$. We prove first that problem (2.17) admits a unique solution $\tilde{\gamma} \in \stackrel{\circ}{W}_{\infty}^{1}(0,1)$. Uniqueness follows from Proposition 2.2. Existence 
can be verified as follows. Let $\varphi$ be the linear functional from $W_{1}^{1}(0,1)$ to $\mathbb{R}$ defined by $\varphi(\chi)=a_{\gamma}\left(u, E_{\gamma} \chi\right)$. Then $\varphi$ is continuous from $W_{1}^{1}(0,1) \rightarrow \mathbb{R}$ in view of $(2.8)$ :

$$
\begin{aligned}
|\varphi(\chi)| & =\left|a_{\gamma}\left(u, E_{\gamma} \chi\right)\right| \leq C\|u\|_{W_{p}^{1}\left(\Omega_{\gamma}\right)}\left\|E_{\gamma} \chi\right\|_{W_{q}^{1}\left(\Omega_{\gamma}\right)} \\
& \leq C \mu\left(\|\gamma\|_{W_{\infty}^{1}(0,1)}\right)\|u\|_{W_{p}^{1}\left(\Omega_{\gamma}\right)}\|\chi\|_{W_{1}^{1}(0,1)} \leq C \varepsilon\|\chi\|_{W_{1}^{1}(0,1)},
\end{aligned}
$$

where the last constant is of the form $C \mu(1 / 2)$. Since $\varphi \in W_{1}^{1}(0,1)^{\prime} \subset$ $W_{2}^{1}(0,1)^{\prime}$, existence of a solution, $\tilde{\gamma}$, in $W_{2}^{1}(0,1)$ is standard; cf. Brezis [7]. The fact that this $\tilde{\gamma}$ resides in $W_{\infty}^{1}(0,1)$ follows from Proposition 2.2 via a density argument. Applying Proposition 2.2 again yields

$$
\begin{aligned}
\|\tilde{\gamma}\|_{W_{\infty}^{1}(0,1)} & \leq \beta \sup _{\substack{0 \neq \chi \in W_{1}^{1}(0,1)\\
}} \frac{b(\tilde{\gamma}, \chi)}{\|\chi\|_{W_{1}^{1}(0,1)}} \\
& =\beta \sup _{\substack{\circ \\
0 \neq \chi \in W_{1}^{1}(0,1)}} \frac{a_{\gamma}\left(u, E_{\gamma}(\chi)\right)}{\|\chi\|_{W_{1}^{1}(0,1)}} \leq C \varepsilon \beta .
\end{aligned}
$$

Choose $\varepsilon$ such that

$$
\varepsilon \leq(2 C \beta)^{-1} \text {. }
$$

Thus, $\|\tilde{\gamma}\|_{W_{\infty}^{1}(0,1)}<1 / 2$.

A similar argument now shows that Proposition 2.3 implies that there is a unique solution $w \in \stackrel{\circ}{W}_{p}^{1}\left(\Omega_{\tilde{\gamma}}\right)$ solving

$$
a_{\tilde{\gamma}}(w, v)=-a_{\tilde{\gamma}}(g, v) \quad \forall v \in \stackrel{\circ}{W}_{q}^{1}\left(\Omega_{\tilde{\gamma}}\right),
$$

and it is bounded by

$$
\|w\|_{W_{p}^{1}\left(\Omega_{\bar{\gamma}}\right)} \leq \alpha \sup _{\substack{0 \neq \hat{v} \in W_{q}^{1}\left(\Omega_{\bar{\gamma}}\right)\\}} \frac{a_{\hat{\gamma}}(g, v)}{\|v\|_{W_{q}^{1}\left(\Omega_{\bar{\gamma}}\right)}} \leq \alpha\|g\|_{W_{p}^{1}\left(\Omega^{*}\right)} .
$$

Therefore, $\tilde{u}=g+w$ satisfies equation (2.18) and

$$
\|\tilde{u}\|_{W_{p}^{1}\left(\Omega_{\bar{\gamma}}\right)} \leq(1+\alpha)\|g\|_{W_{p}^{1}\left(\Omega^{*}\right)} .
$$

Now choose $\delta$ such that $\delta \leq \varepsilon /(1+\alpha)$. Hence, $\|g\|_{W_{p}^{1}\left(\Omega^{*}\right)}<\delta$ implies $(\tilde{\gamma}, \tilde{u}) \in$ $V_{\varepsilon}$.

Although the set $V_{\varepsilon}$ is appropriate from a physical point of view, it is not convenient for measuring the difference between two solutions because it is not based on a linear space. For this reason, we introduce the set

$$
\widehat{V}_{\varepsilon}=\left\{(\gamma, \hat{u}) \in \stackrel{\circ}{W}_{\infty}^{1}(0,1) \times \stackrel{\circ}{W}_{p}^{1}\left(\Omega_{0}\right):\|\gamma\|_{W_{\infty}^{1}(0,1)}<\frac{1}{2},\|\hat{u}\|_{W_{p}^{1}\left(\Omega_{0}\right)}<\varepsilon\right\} .
$$

There is a natural mapping between $V_{\varepsilon}$ and $\widehat{V}_{\varepsilon}$ induced by (2.3), namely $(\gamma, v) \rightarrow(\gamma, \hat{v})$, where $\hat{v}$ is defined by (2.4). The inverse mapping is well defined in view of (2.6). A mapping $\widehat{T}$ can be defined correspondingly via

$$
\widehat{T}(\gamma, \hat{v}):=\left(T_{1}(\gamma, v), T_{2} \widehat{(\gamma, v)}\right) .
$$


However, note that the " $\sim$ " on $v$ refers to the mapping (2.3) generated by $\gamma$, whereas the one on $T_{2}(\gamma, v)$ refers to the one generated by $\tilde{\gamma}=T_{1}(\gamma, v)$. The result is a mapping related to $T$ that maps a subset of a linear space, $\widehat{V}_{\varepsilon}$, into itself.

Theorem 2.1. Let $P$ and $\delta$ be as in Lemma 2.1. For all $p \in(2, P)$ and all $g \in W_{p}^{1}\left(\Omega^{*}\right)$ such that $\|g\|_{W_{p}^{1}\left(\Omega^{*}\right)}<\delta$, the mapping $\widehat{T}$ is a contraction from $\widehat{V}_{\varepsilon} \rightarrow \widehat{V}_{\varepsilon}$ with respect to the norm

$$
\left\|\left|(\gamma, \hat{u})\|\mid=\varepsilon\| \gamma\left\|_{W_{\infty}^{1}(0,1)}+\right\| \hat{u} \|_{W_{p}^{1}\left(\Omega_{0}\right)}\right.\right.
$$

for $\varepsilon$ sufficiently small.

Proof. By Lemma 1.1 we have that $\widehat{T}: \widehat{V}_{\varepsilon} \rightarrow \widehat{V}_{\varepsilon}$ for $\varepsilon$ sufficiently small. In order to prove that $\hat{T}$ is a contraction, take $\tilde{\gamma}^{j}=T_{1}\left(\gamma^{j}, u^{j}\right)$ and $\tilde{u}^{j}=T_{2}\left(\gamma^{j}, u^{j}\right)$, where $\left(\gamma^{j}, u^{j}\right)$ is any element of $V_{\varepsilon}, j=1,2$. Let $\gamma=\tilde{\gamma}^{1}-\tilde{\gamma}^{2}$. Then $\gamma \in \stackrel{\circ}{W}_{\infty}^{1}(0,1)$, and for all $\chi \in \stackrel{\circ}{W}_{1}^{1}(0,1), \gamma$ satisfies

$$
\begin{aligned}
b(\gamma, \chi)= & b\left(\tilde{\gamma}^{1}-\tilde{\gamma}^{2}, \chi\right)=\hat{a}\left(\hat{u}^{1}, \hat{E} \chi ; \gamma^{1}\right)-\hat{a}\left(\hat{u}^{2}, \hat{E} \chi ; \gamma^{2}\right) \\
= & \int_{\Omega_{0}} \sum_{i, j=1}^{2} A_{i j}\left(\xi, \eta ; \gamma^{1}\right) \partial_{j} \hat{u}^{1} \partial_{i} \hat{E} \chi d \xi d \eta \\
& -\int_{\Omega_{0}} \sum_{i, j=1}^{2} A_{i j}\left(\xi, \eta ; \gamma^{2}\right) \partial_{j} \hat{u}^{2} \partial_{i} \hat{E} \chi d \xi d \eta \\
= & \int_{\Omega_{0}} \sum_{i, j=1}^{2}\left[A_{i j}\left(\xi, \eta ; \gamma^{1}\right)-A_{i j}\left(\xi, \eta ; \gamma^{2}\right)\right] \partial_{j} \hat{u}^{1} \partial_{i} \hat{E} \chi d \xi d \eta \\
& +\int_{\Omega_{0}} \sum_{i, j=1}^{2} A_{i j}\left(\xi, \eta ; \gamma^{2}\right)\left[\partial_{j} \hat{u}^{1}-\partial_{j} \hat{u}^{2}\right] \partial_{i} \widehat{E} \chi d \xi d \eta .
\end{aligned}
$$

In view of (2.6) and (2.13), there exists $C<\infty$ such that

$$
\begin{aligned}
|b(\gamma, \chi)| \leq & C\left[\left\|\hat{u}^{1}\right\|_{W_{p}^{1}\left(\Omega_{0}\right)}\left\|\gamma^{1}-\gamma^{2}\right\|_{W_{\infty}^{1}(0,1)}\right. \\
& \left.+\left\|\gamma^{2}\right\|_{W_{\infty}^{1}(0,1)}\left\|\hat{u}^{1}-\hat{u}^{2}\right\|_{W_{p}^{1}\left(\Omega_{0}\right)}\right]\|\chi\|_{W_{1}^{1}(0,1)} \\
\leq & C\left[\varepsilon\left\|\gamma^{1}-\gamma^{2}\right\|_{W_{\infty}^{1}(0,1)}+\left\|\hat{u}^{1}-\hat{u}^{2}\right\|_{W_{p}^{1}\left(\Omega_{0}\right)}\right]\|\chi\|_{W_{1}^{1}(0,1)},
\end{aligned}
$$

since $\left(\gamma^{1}, u^{1}\right) \in V_{\varepsilon}$ and $\left(\gamma^{2}, u^{2}\right) \in V_{\varepsilon}$ implies that $\left\|\gamma^{2}\right\|_{W_{\infty}^{1}(0,1)}<1 / 2$ and $\left\|\hat{u}^{1}\right\|_{W_{p}^{1}\left(\Omega_{0}\right)}<\varepsilon$. From Proposition 2.2, we conclude that

$$
\|\gamma\|_{W_{\infty}^{1}(0,1)} \leq C \beta\left(\varepsilon\left\|\gamma^{1}-\gamma^{2}\right\|_{W_{\infty}^{1}(0,1)}+\left\|\hat{u}^{1}-\hat{u}^{2}\right\|_{W_{p}^{1}\left(\Omega_{0}\right)}\right) .
$$


To bound the difference between $\tilde{u}^{1}$ and $\tilde{u}^{2}$, we do the following. Note first that $\widehat{\tilde{u}^{1}}$ and $\widehat{\tilde{u}^{2}}$ satisfy

$$
\begin{array}{ll}
\hat{a}\left(\hat{u}^{1}, \hat{v} ; \tilde{\gamma}^{1}\right)=0 & \forall \hat{v} \in \stackrel{\circ}{W}_{q}^{1}\left(\Omega_{0}\right), \\
\hat{a}\left(\widehat{\tilde{u}^{2}}, \hat{v} ; \tilde{\gamma}^{2}\right)=0 & \forall \hat{v} \in \stackrel{\circ}{W}_{q}^{1}\left(\Omega_{0}\right) .
\end{array}
$$

Because we assumed that $g(x, y)=0$ for $y \geq 1 / 2$ and $\tilde{\gamma}^{j}(i)=0$ for $i=0,1$ and $j=1,2$, we have $\hat{g}^{1}-\hat{g}^{2} \in \stackrel{\circ}{W}_{p}^{1}\left(\Omega_{0}\right)$, where the different "hats" refer to different mappings (2.3). Thus, $\hat{u}:=\widehat{\tilde{u}^{1}}-\widehat{\tilde{u}^{2}} \in \stackrel{\circ}{W}_{p}^{1}\left(\Omega_{0}\right)$ and satisfies

$$
\begin{aligned}
\hat{a}\left(\hat{u}, \hat{v} ; \tilde{\gamma}^{1}\right) & =\hat{a}\left(\widehat{\tilde{u}^{1}}, \hat{v} ; \tilde{\gamma}^{1}\right)-\hat{a}\left(\widehat{\tilde{u}^{2}}, \hat{v} ; \tilde{\gamma}^{1}\right) \\
& =-\hat{a}\left(\widehat{\tilde{u}^{2}}, \hat{v} ; \tilde{\gamma}^{1}\right)=\hat{a}\left(\widehat{\tilde{u}^{2}}, \hat{v} ; \tilde{\gamma}^{2}\right)-\hat{a}\left(\widehat{\tilde{u}^{2}}, \hat{v} ; \tilde{\gamma}^{1}\right) \\
& =\int_{\Omega_{0}} \sum_{i, j=1}^{2}\left[A_{i j}\left(\xi, \eta ; \tilde{\gamma}^{2}\right)-A_{i j}\left(\xi, \eta ; \tilde{\gamma}^{1}\right)\right] \partial_{j} \widehat{\tilde{u}^{2}} \partial_{i} \hat{v} d \xi d \eta .
\end{aligned}
$$

Therefore,

$$
\begin{aligned}
\left|\hat{a}\left(\hat{u}, \hat{v} ; \tilde{\gamma}^{1}\right)\right| & \leq C\left\|\tilde{\gamma}^{1}-\tilde{\gamma}^{2}\right\|_{W_{\infty}^{1}(0,1)}\left\|\widehat{\tilde{u}^{2}}\right\|_{W_{p}^{1}\left(\Omega_{0}\right)}\|\hat{v}\|_{W_{q}^{1}\left(\Omega_{0}\right)} \\
& \leq C \varepsilon\left\|\tilde{\gamma}^{1}-\tilde{\gamma}^{2}\right\|_{W_{\infty}^{1}(0,1)}\|\hat{v}\|_{W_{q}^{1}\left(\Omega_{0}\right)} \\
& =C \varepsilon\|\gamma\|_{W_{\infty}^{1}(0,1)}\|\hat{v}\|_{W_{q}^{1}\left(\Omega_{0}\right)} .
\end{aligned}
$$

Inequality (2.16) then yields

$$
\|\hat{u}\|_{W_{p}^{1}\left(\Omega_{0}\right)} \leq \alpha \sup _{\substack{0 \neq \hat{v} \in W_{q}^{1}\left(\Omega_{0}\right)\\}} \frac{\hat{a}\left(\hat{u}, \hat{v} ; \tilde{\gamma}^{1}\right)}{\|\hat{v}\|_{W_{q}^{1}\left(\Omega_{0}\right)}} \leq C \varepsilon \alpha\|\gamma\|_{W_{\infty}^{1}(0,1)}
$$

Applying inequality (2.21) gives

$$
\|\hat{u}\|_{W_{p}^{1}\left(\Omega_{0}\right)} \leq C \alpha \beta \varepsilon\left[\varepsilon\left\|\gamma^{1}-\gamma^{2}\right\|_{W_{\infty}^{1}(0,1)}+\left\|\hat{u}^{1}-\hat{u}^{2}\right\|_{W_{p}^{1}\left(\Omega_{0}\right.}\right] .
$$

Inserting this inequality and inequality (2.21) in the definition of $\||\cdot|\|$, we have

$$
\left\|\widehat{T}\left(\gamma^{1}, \widehat{u^{1}}\right)-\widehat{T}\left(\gamma^{2}, \widehat{u^{2}}\right)\left|\|\leq C(1+\alpha) \beta \varepsilon\|\left\|\left(\gamma^{1}, \widehat{u^{1}}\right)-\left(\gamma^{2}, \widehat{u^{2}}\right)\right\|\right|\right.
$$

Choosing $\varepsilon<(C(1+\alpha) \beta)^{-1}$ we conclude that $\widehat{T}$ is a contraction from $\widehat{V}_{\varepsilon} \rightarrow$ $\widehat{V}_{\varepsilon}$.

Corollary 2.1. If $\|g\|_{W_{p}^{1}\left(\Omega^{*}\right)}$ is sufficiently small, then for all $p \in(2, P)$ problem (2.9) admits a unique solution $(\gamma, u) \in V_{\varepsilon}$ which can be calculated by a fixed 
point iteration,

$$
\left(\gamma^{i+1}, u^{i+1}\right)=T\left(\gamma^{i}, u^{i}\right),
$$

starting with any $\left(\gamma^{0}, u^{0}\right) \in V_{\varepsilon}$. of

One choice of $\gamma^{0}$ and $u^{0}$ can be the following: $\gamma^{0} \equiv 0$ and $u^{0}$ the solution

$$
\begin{aligned}
\Delta u=0 & \text { in } \Omega_{0}, \\
u=g & \text { on } \partial \Omega_{0} .
\end{aligned}
$$

Clearly, $\left\|u^{0}\right\|_{W_{p}^{1}\left(\Omega_{0}\right)} \leq C\|g\|_{W_{p}^{1}\left(\Omega^{*}\right)}$, so choosing $\delta$ as was required in the proof of Lemma 2.1 yields $\left(\gamma^{0}, u^{0}\right) \in V_{\varepsilon}$.

We conclude this section with some remarks regarding its results.

(1) We have not proved global uniqueness, and there may indeed be solutions of larger norm even for small data.

(2) Regularity of solutions can be studied using the techniques in the works cited at the beginning that use Hölder norms. The solutions obtained by those techniques, when applicable, are of course identical to the weak solutions obtained here.

\section{THE VARIATIONAL FORMULATION OF THE DISCRETE PROBLEM}

Let $\pi_{h}^{0}, 0<h \leq h_{0}<1$, be a quasi-uniform triangulation of $\Omega_{0}$, i.e., such that the following regularity condition is satisfied: the triangles $\widetilde{K} \in \pi_{h}^{0}$ meet only in entire common sides or in vertices; each triangle $\widetilde{K} \in \pi_{h}^{0}$ contains a circle of radius $c_{1} h$ and is contained in a circle of radius $c_{2} h$, where the constants $c_{1}>0$ and $c_{2}<\infty$ do not depend on $\widetilde{K}$ or $h$.

We denote by $\pi_{h}^{\gamma}$ the triangulation obtained by transforming the vertices of $\pi_{h}^{0}$ via the mapping in (2.3). More precisely, let $F^{\gamma}$ denote the continuous, piecewise affine interpolant of the mapping (2.3) with respect to the mesh $\pi_{h}^{0}$, and let $\pi_{h}^{\gamma}$ be the image of $\pi_{h}^{0}$ with respect to $F^{\gamma}$. Denote by $\Omega_{\gamma}^{h}$ the image of $\Omega_{0}$ with respect to $F^{\gamma}$. For each triangle $\widetilde{K}$ in $\pi_{h}^{0}$, denote its vertices by $\tilde{\mathbf{n}}_{K, j}=\left(\xi_{K, j}, \eta_{K, j}\right)$ for $j=1,2,3$, and let $\mathbf{n}_{K, j}:=F^{\gamma}\left(\tilde{\mathbf{n}}_{K, j}\right)$. The mapping $F^{\gamma}$ is given on each triangle by

$$
\left.F^{\gamma}\right|_{\widetilde{K}}(\xi, \eta)=D_{K}(\xi, \eta)+\vec{d}_{K} \quad \forall(\xi, \eta) \in \widetilde{K},
$$

where $D_{K}$ is a $2 \times 2$ matrix and $\vec{d}_{K} \in \mathbb{R}^{2}$. When it is not confusing to do so, we shall refer to the vertices by a global node number $k=k(K, j)$.

Proposition 3.1. If $\gamma \in W_{\infty}^{1}(0,1)$ and $\|\gamma\|_{W_{\infty}^{1}(0,1)}<1$, then the mapping $F^{\gamma}$ is invertible and $\pi_{h}^{\gamma}$ is a quasi-uniform triangulation. Furthermore, the entries of the Jacobians, $D_{K}$, of $\left.F^{\gamma}\right|_{\widetilde{K}}$, together with their inverses, are all bounded by $C\left(1-\|\gamma\|_{W_{\infty}^{1}(0,1)}\right)^{-1}$. 
Proof. Let $c=\|\gamma\|_{W_{\infty}^{1}(0,1)}<1$. Let $k=k(K, j)$ and $k^{\prime}=k^{\prime}\left(K, j^{\prime}\right)$ denote node indices of two distinct vertices $\left(j \neq j^{\prime}\right)$ of a given triangle. We have

$$
\begin{aligned}
\left|y_{k}-y_{k^{\prime}}\right| & =\left|\eta_{k}-\eta_{k^{\prime}}+\gamma\left(\xi_{k}\right) \eta_{k}-\gamma\left(\xi_{k^{\prime}}\right) \eta_{k^{\prime}}\right| \\
& =\left|\left(\eta_{k}-\eta_{k^{\prime}}\right)\left(1+\gamma\left(\xi_{k^{\prime}}\right)\right)+\eta_{k}\left(\gamma\left(\xi_{k}\right)-\gamma\left(\xi_{k^{\prime}}\right)\right)\right| \\
& \geq\left|\left(\eta_{k}-\eta_{k^{\prime}}\right)\left(1+\gamma\left(\xi_{k^{\prime}}\right)\right)\right|-\left|\eta_{k}\left(\gamma\left(\xi_{k}\right)-\gamma\left(\xi_{k^{\prime}}\right)\right)\right| \\
& \geq(1-c)\left|\eta_{k}-\eta_{k^{\prime}}\right|-c\left|\eta_{k}\left(\xi_{k}-\xi_{k^{\prime}}\right)\right| \\
& \geq(1-c)\left|\eta_{k}-\eta_{k^{\prime}}\right|-c\left|\xi_{k}-\xi_{k^{\prime}}\right| .
\end{aligned}
$$

Since $x_{k}=\xi_{k}$, we have

$$
\left|x_{k}-x_{k^{\prime}}\right|+\left|y_{k}-y_{k^{\prime}}\right| \geq(1-c)\left(\left|\eta_{k}-\eta_{k^{\prime}}\right|+\left|\xi_{k}-\xi_{k^{\prime}}\right|\right) .
$$

Similarly, we can estimate that

$$
\left|x_{k}-x_{k^{\prime}}\right|+\left|y_{k}-y_{k^{\prime}}\right| \leq(1+c)\left(\left|\eta_{k}-\eta_{k^{\prime}}\right|+\left|\xi_{k}-\xi_{k^{\prime}}\right|\right) \text {. }
$$

Thus each triangle is mapped onto a triangle of comparable dimensions, so that $\pi_{h}^{\gamma}$ is a quasi-uniform triangulation. Moreover, this also shows that each $F_{i}^{\gamma}$, and hence $F^{\gamma}$, is invertible.

Since $F^{\gamma}\left(\tilde{\mathbf{n}}_{i, 3}\right)=\mathbf{n}_{i, 3}$, we may write

$$
\left.F^{\gamma}\right|_{\widetilde{K}}\left((\xi, \eta)+\tilde{\mathbf{n}}_{i, 3}\right)=D_{K}(\xi, \eta)+\mathbf{n}_{i, 3} .
$$

Since $F^{\gamma}\left(\tilde{\mathbf{n}}_{i, j}\right)=\mathbf{n}_{i, j}$ for $j=1,2$, we must have

$$
D_{K} \tilde{C}^{j}=C^{j} \quad \forall j=1,2,
$$

where $C^{j}:=\mathbf{n}_{i, j}-\mathbf{n}_{i, 3}$ and $\widetilde{C}^{j}:=\tilde{\mathbf{n}}_{i, j}-\tilde{\mathbf{n}}_{i, 3}$. If we let $C$ (resp. $\widetilde{C}$ ) denote the matrix with columns $C^{j}$ (resp. $\tilde{C}^{j}$ ), then we have $D_{K} \widetilde{C}=C$ or $D_{K}=C \tilde{C}^{-1}$. Thus, we also have $D_{K}^{-1}=\widetilde{C} C^{-1}$. From these representations and the bounds above, the claimed estimates on the entries of $D_{K}$ and $D_{K}^{-1}$ follow easily.

As a consequence of Proposition 3.1, if $\|\gamma\|_{W_{\infty}^{1}(0,1)}<1$, then the mapping $F^{\gamma}$ induces an isomorphism, $v \rightarrow \tilde{v}:=v \circ F^{\gamma}$, of the spaces $W_{q}^{1}\left(\Omega_{\gamma}^{h}\right) \rightarrow W_{q}^{1}\left(\Omega_{0}\right)$. Moreover, we have the estimates

$$
\begin{aligned}
\|v\|_{W_{q}^{1}\left(\Omega_{\gamma}^{h}\right)} & \leq \mu\left(\|\gamma\|_{W_{\infty}^{1}(0,1)}\right)\|\tilde{v}\|_{W_{q}^{1}\left(\Omega_{0}\right)}, \quad 1 \leq q \leq \infty . \\
\|\tilde{v}\|_{W_{q}^{1}\left(\Omega_{0}\right)} \leq \mu\left(\|\gamma\|_{W_{\infty}^{1}(0,1)}\right)\|v\|_{W_{q}^{1}\left(\Omega_{\gamma}^{h}\right)}, &
\end{aligned}
$$

Note that the transformation $\Omega_{0} \rightarrow \Omega_{\gamma}^{h}$ is different from the change of variables (2.3) that we used in the continuous case; to distinguish one from the other, we denote by $(\tilde{x}, \tilde{y})$ the point in $\Omega_{0}$ related to $(x, y) \in \Omega_{\gamma}^{h}$ by

$$
F^{\gamma}(\tilde{x}, \tilde{y})=(x, y),
$$

whereas we denoted $(2.3)$ via $(\hat{x}, \hat{y}) \rightarrow(x, y)$. The transformation $F^{\gamma}$ is introduced in order to transform the triangles $\widetilde{K}$ into triangles $K$ and the space $\mathbf{P}^{1}(\widetilde{K})$ into $\mathbf{P}^{1}(K)$. This would no longer be true if we applied the transformation (2.3) to the triangulation $\pi_{h}^{0}$. 
Related to the triangulations $\pi_{h}^{0}$ and $\pi_{h}^{\gamma}$, respectively, we define the following spaces:

$$
\begin{aligned}
& V_{h}^{\gamma}=\left\{v \in C^{0}\left(\Omega_{\gamma}^{h}\right):\left.v\right|_{K} \in \mathbf{P}^{1}(K) \quad \forall K \in \pi_{h}^{\gamma}\right\}, \\
& \stackrel{\circ}{V}_{h}^{\gamma}=\left\{v \in V_{h}^{\gamma}: v\left(\mathbf{n}_{i}\right)=0 \quad \forall \mathbf{n}_{i} \in \partial \Omega_{\gamma}^{h}\right\}, \\
& V_{h}^{0}=\left\{\tilde{v} \in C^{0}\left(\Omega_{0}\right):\left.\tilde{v}\right|_{\widetilde{K}} \in \mathbf{P}^{1}(\widetilde{K}) \quad \forall \tilde{K} \in \pi_{h}^{0}\right\}, \\
& \stackrel{\circ}{V}_{h}^{0}=\left\{\tilde{v} \in V_{h}^{0}: \tilde{v}\left(\tilde{\mathbf{n}}_{i}\right)=0 \quad \forall \tilde{\mathbf{n}}_{i} \in \partial \Omega_{0}\right\} .
\end{aligned}
$$

Let $\left\{\xi_{i} \in[0,1]: 1 \leq i \leq l_{h}\right\}$ denote all $x$-coordinates such that $\left(\xi_{i}, 1\right)$ is a vertex of a triangle $\widetilde{K} \in \pi_{h}^{0}$, i.e., $\xi_{i}=\xi_{k}$ for some $k$. Decompose $[0,1]=$ $\bigcup_{i=1}^{l_{h}}\left[\xi_{i-1}, \xi_{i}\right]$, and relate to this mesh the following discrete space:

$$
\begin{aligned}
S_{h}=\left\{\gamma \in C^{0}(0,1):\left.\gamma\right|_{\left[\xi_{i-1}, \xi_{i}\right]} \in \mathbf{P}^{1}\left(\left[\xi_{i-1}, \xi_{i}\right]\right),\right. & 0 \leq i \leq l_{h}+1, \\
& \text { and } \gamma(0)=\gamma(1)=0\} .
\end{aligned}
$$

These are the spaces to be used in the variational approximation of (2.9).

Piecewise linear interpolants. Given $\gamma \in \stackrel{\circ}{W}_{\infty}^{1}(0,1), 1 \leq p \leq \infty$, we define $I_{h} \gamma$ to be the usual piecewise linear interpolant, based on the knots $\left\{\xi_{i}: 1 \leq i \leq l_{h}\right\}$, from $C^{0}(0,1)$ to $\left\{\gamma \in C^{0}(0,1):\left.\gamma\right|_{\left[\xi_{i-1}, \xi_{i}\right]} \in \mathbf{P}^{1}\left(\left[\xi_{i-1}, \xi_{i}\right]\right)\right\}$. If $\gamma \in W_{\infty}^{2}(0,1)$ $\cap \stackrel{\circ}{W}_{\infty}^{1}(0,1)$, the following estimate holds:

$$
\left\|\gamma-I_{h} \gamma\right\|_{W_{\infty}^{1}(0,1)} \leq C h\|\gamma\|_{W_{\infty}^{2}(0,1)} .
$$

For functions $v \in W_{p}^{1}\left(\Omega_{\gamma}^{h}\right)$ with $p>2$ we shall use the usual piecewise linear interpolant $I_{h}^{\gamma}: W_{p}^{1}\left(\Omega_{\gamma}^{h}\right) \rightarrow V_{h}^{\gamma}$, namely

$$
I_{h}^{\gamma} v=\sum_{k=1}^{m_{h}} v\left(\mathbf{n}_{k}\right) v_{k}^{h},
$$

where $\left\{v_{k}^{h}: 1 \leq k \leq m_{h}\right\}$ is the usual Lagrange basis of $V_{h}^{\gamma}$ defined by

$$
v_{k}^{h}\left(\mathbf{n}_{k^{\prime}}\right)=\delta_{k k^{\prime}}, \quad 1 \leq k, k^{\prime} \leq m_{h} \text {. }
$$

Note that, for any $v \in W_{p}^{1}\left(\Omega_{\gamma}^{h}\right)$,

$$
\widetilde{I_{h}^{\gamma} v}=I_{h}^{0} \hat{v}=I_{h}^{0} \tilde{v} .
$$

As usual, we have

$$
\left\|w-I_{h}^{0} w\right\|_{W_{p}^{1}\left(\Omega_{0}\right)} \leq C h\|w\|_{W_{p}^{2}\left(\Omega_{0}\right)} .
$$

Also note that $I_{h}\left(\left.v\right|_{\{(x, 1): 0 \leq x \leq 1\}}\right)=\left.\left(I_{h}^{0} v\right)\right|_{\{(x, 1): 0 \leq x \leq 1\}}$, so our notations for the boundary and interior interpolants do not conflict. Finally, let $g_{h}^{\gamma}=I_{h}^{\gamma} g$; if $g \in W_{p}^{2}\left(\Omega^{*}\right)$ for $p>2$, then

$$
\left\|g_{h}^{\gamma}\right\|_{W_{p}^{1}\left(\Omega_{\gamma}^{h}\right)} \leq C\|g\|_{W_{p}^{1}\left(\Omega^{*}\right)} .
$$


Let $\chi_{h} \in S_{h}$. As $\chi_{h} \in W_{p}^{1}(0,1)$, for $1 \leq p \leq \infty$, we can consider the extension, $E \chi_{h}$, of $\chi_{h}$ to $\Omega_{0}$ defined in the proof of Lemma 2.0. We know from the continuous case, $(2.2)$, that $E \chi_{h} \in W_{q}^{1}\left(\Omega_{0}\right)$ for $q<2$. In order to construct a piecewise linear interpolant of this extension to $V_{h}^{0}$, we shall use a variant of a piecewise linear interpolant for "rough functions," which was introduced by Clement [9]. The presentation that appears in Scott and Zhang [25] will be used. -They show that there is an interpolant, $J_{h}$, that is given by

$$
J_{h}^{\gamma} v=\sum_{k=1}^{m_{h}} L_{k}(v) v_{k}^{h}
$$

where $L_{k}$ is a linear functional given by a weighted integral along an edge in the triangulation having $\mathbf{n}_{k}$ as a node. We can pick these edges arbitrarily, so that for all boundary nodes, $\mathbf{n}_{k} \in\{(x, 1): 0 \leq x \leq 1\}$, we can require all the edges to lie on the boundary segment $\{(x, 1): 0 \leq x \leq 1\}$. Moreover, $J_{h}$ is a projection that reproduces any piecewise linear function locally; in particular, $J_{h} E \chi$ is an extension of $\chi$ to $V_{h}^{0}$. Finally, it is shown in [25] that

$$
\begin{aligned}
\left\|J_{h} v\right\|_{W_{q}^{1}\left(\Omega_{0}\right)} & \leq C\|v\|_{W_{q}^{1}\left(\Omega_{0}\right)}, \\
\left\|v-J_{h} v\right\|_{W_{q}^{1}\left(\Omega_{0}\right)} & \leq C h\|v\|_{W_{q}^{2}\left(\Omega_{0}\right)},
\end{aligned}
$$

where $\mathrm{C}$ is a constant independent of $h$ or $v$, and $1 \leq q \leq \infty$. Now we can define the "discrete extension" $E_{\gamma}^{h} \chi_{h}$ of $\chi_{h}$ to the whole domain $\Omega_{\gamma}^{h}$ by

$$
\widetilde{E_{\gamma}^{h} \chi_{h}}=J_{h} E \chi_{h} \text {. }
$$

Clearly, $E_{\gamma}^{h} \chi_{h} \in V_{h}^{\gamma}$, and from inequalities (3.7) and (3.1) we can assert the existence of a positive constant $C$, independent of $h$ and $\chi_{h}$, such that

$$
\left\|E_{\gamma}^{h} \chi_{h}\right\|_{W_{q}^{1}\left(\Omega_{\gamma}^{h}\right)} \leq C \mu\left(\|\gamma\|_{W_{\infty}^{1}(0,1)}\right)\left\|\chi_{h}\right\|_{W_{1}^{1}(0,1)} .
$$

The discrete free-boundary problem. We pose the following discrete problem associated with the quasi-uniform triangulation $\pi_{h}^{0}$.

Find $\gamma_{h} \in S_{h}$ and $u_{h} \in g_{h}^{\gamma_{h}} \oplus V_{h}^{\gamma_{h}}$ such that

$$
\begin{gathered}
a_{\gamma_{h}}\left(u_{h}, v_{h}\right)=0 \quad \forall v_{h} \in \stackrel{\circ}{V}_{h}^{\gamma_{h}}, \\
b\left(\gamma_{h}, \chi\right)=a_{\gamma_{h}}\left(u_{h}, E_{\gamma_{h}}^{h} \chi\right) \quad \forall \chi \in S_{h} .
\end{gathered}
$$

Remark. In view of (3.9a), the right-hand side of equation (3.9b) can be written as

$$
a_{\gamma_{h}}\left(u_{h}, E_{\gamma_{h}}^{h} \chi\right)=a_{\gamma_{h}}\left(u_{h}, E_{h} \chi\right)
$$

for any extension $E_{h}$, e.g., we can take

$$
E_{h} \chi=\sum_{\mathbf{n}_{k} \in\{(x, 1): 0 \leq x \leq 1\}} \chi\left(\xi_{k}\right) v_{k}^{h},
$$


since $E_{h} \chi-E_{\gamma}^{h} \chi \in \stackrel{\circ}{W}_{q}^{1}\left(\Omega_{\gamma}\right)$. Thus, the right-hand side of equation (3.9b) can be easily computed.

To prove the existence of a solution of the discrete problem (3.9), we need to prove a discrete version of Propositions 2.2 and 2.3.

Proposition 3.2. There exists a positive constant $\beta$, independent of $h$, such that for any $\gamma_{h} \in S_{h}$

$$
\left\|\gamma_{h}\right\|_{W_{\infty}^{1}(0,1)} \leq \beta \sup _{0 \neq \chi_{h} \in S_{h}} \frac{b\left(\gamma_{h}, \chi_{h}\right)}{\left\|\chi_{h}\right\|_{W_{1}^{1}(0,1)}} .
$$

Proof. Let $P_{h}$ denote the projection from $W_{1}^{1}(0,1)$ to $S_{h}$ defined by

$$
b\left(P_{h} \chi-\chi, \sigma\right)=0 \quad \forall \sigma \in S_{h} .
$$

The results of Douglas, Dupont, and Wahlbin [11] imply that for any $\chi \in$ $W_{p}^{1}(0,1)$

$$
\left\|P_{h} \chi\right\|_{W_{p}^{1}(0,1)} \leq C\|\chi\|_{W_{p}^{1}(0,1)}, \quad 1 \leq p \leq \infty .
$$

For $\gamma_{h} \in \stackrel{\circ}{W}_{\infty}^{1}(0,1)$, inequalities $(2.10)$ and (3.11) with $p=1$ imply that

$$
\begin{aligned}
\left\|\gamma_{h}\right\|_{W_{\infty}^{1}(0,1)} & \leq \beta \sup _{\substack{\circ \\
0 \neq \chi \in W_{1}^{1}(0,1)}} \frac{b\left(\gamma_{h}, \chi\right)}{\|\chi\|_{W_{1}^{1}(0,1)}}=\beta \sup _{\substack{\circ \\
0 \neq \chi \in W_{1}^{1}(0,1)}} \frac{b\left(\gamma_{h}, P_{h} \chi\right)}{\|\chi\|_{W_{1}^{1}(0,1)}} \\
& \leq C \beta \sup _{\substack{0 \neq \chi \in W_{1}^{1}(0,1)\\
}} \frac{b\left(\gamma_{h}, P_{h} \chi\right)}{\left\|P_{h} \chi\right\|_{W_{1}^{1}(0,1)}}=C \beta \sup _{0 \neq \chi_{h} \in S_{h}} \frac{b\left(\gamma_{h}, \chi_{h}\right)}{\left\|\chi_{h}\right\|_{W_{1}^{1}(0,1)}} .
\end{aligned}
$$

Choosing the new $\beta=C \beta$ gives us inequality (3.10).

We now prove an inequality analogous to $(2.11)$ for the discrete case. Since this result is a special case of a result of independent interest, namely a stability bound for the Ritz projection with respect to a bilinear form having only bounded, measurable coefficients, we give the more general version first.

Proposition 3.3. Suppose that $\Omega_{0}$ is a convex polygon and that the bilinear form

$$
a(u, v)=\int_{\Omega_{0}} \sum_{i, j=1}^{2} A_{i j}(\xi, \eta) \partial_{i} u \partial_{j} v d \xi d \eta
$$

satisfies the conditions (2.14) and (2.15). Let $\stackrel{\circ}{V}_{h}^{0}$ denote piecewise linear functions, on any quasi-uniform mesh, that vanish on the boundary. Then there are constants $\alpha<\infty, h_{0}>0$ and $2<P$ such that for all $0<h \leq h_{0}$ and $u_{h} \in \stackrel{\circ}{V}_{h}^{0}$

$$
\left\|u_{h}\right\|_{W_{p}^{1}\left(\Omega_{0}\right)} \leq \alpha \sup _{0 \neq v_{h} \in V_{h}^{0}} \frac{a\left(u_{h}, v_{h}\right)}{\left\|v_{h}\right\|_{W_{q}^{1}\left(\Omega_{0}\right)}},
$$

whenever $2 \leq p \leq P$ ( $q$ is the dual index to $p$, i.e., $q=p / p-1$ ). 
Let $R_{h} u$ be the Ritz projection with respect to the bilinear form $a(u, v)$ of an element $u \in \stackrel{\circ}{W}_{2}^{1}\left(\Omega_{0}\right)$ on $\stackrel{\circ}{V}_{h}^{0}$, i.e., $R_{h} u$ is the unique element of $\stackrel{\circ}{V}_{h}^{0}$ which satisfies

$$
a\left(u-R_{h} u, v\right)=0 \quad \forall v \in \stackrel{\circ}{W}_{2}^{1}\left(\Omega_{0}\right) .
$$

Corollary 3.0. Under the assumptions of Proposition 3.3, the Ritz projection is stable in $W_{p}^{1}\left(\Omega_{0}\right)$, i.e., there is a positive constant $C$, independent of $h$ and $u$, such that

$$
\left\|R_{h} u\right\|_{W_{p}^{1}\left(\Omega_{0}\right)} \leq C\|u\|_{W_{p}^{1}\left(\Omega_{0}\right)}, \quad 2 \leq p \leq P .
$$

This follows from Proposition 3.3 by applying the definition of $R_{h}$ and Hölder's inequality:

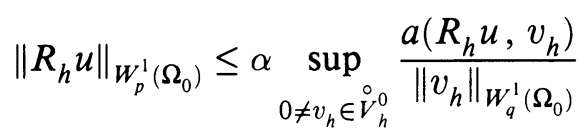

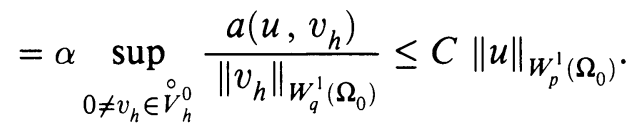

In view of the discussion in the proof of Proposition 2.3, the following is an immediate consequence of Proposition 3.3.

Proposition 3.4. Suppose $\|\gamma\|_{W_{\infty}^{1}(0,1)}<1 / 2$. Then there are constants $\alpha<\infty$ and $2<P$, independent of $h$, such that for all $u_{h} \in \stackrel{\circ}{V}_{h}^{0}$

$$
\left\|u_{h}\right\|_{W_{p}^{1}\left(\Omega_{0}\right)} \leq \alpha \sup _{\substack{0 \neq v_{h} \in V_{h}^{0}\\}} \frac{\hat{a}\left(u_{h}, v_{h} ; \gamma\right)}{\left\|v_{h}\right\|_{W_{q}^{1}\left(\Omega_{0}\right)}},
$$

whenever $2 \leq p \leq P$.

Proof of Proposition 3.3. The proof is based on the ideas of Meyers [17]. First we establish the corresponding inequality in the case that the bilinear form in question is much simpler. Consider the Ritz projection $R_{h}^{*}: \stackrel{\circ}{W}_{2}^{1} \rightarrow \stackrel{\circ}{V}_{h}^{0}$ defined by

$$
\left\langle\nabla\left(R_{h}^{*} u-u\right), \nabla v_{h}\right\rangle=0 \quad \forall v_{h} \in \stackrel{\circ}{V}_{h}^{0},
$$

where $\langle\cdot, \cdot\rangle$ denotes the vector- $L^{2}$ inner product on $\Omega_{0}$. Rannacher and Scott [21] proved that

$$
\left\|R_{h}^{*} u\right\|_{\stackrel{\circ}{p}_{p}^{1}\left(\Omega_{0}\right)} \leq C^{*}\|u\|_{\dot{W}_{p}^{1}\left(\Omega_{0}\right)}, \quad 2 \leq p \leq \infty .
$$

An argument similar to (3.12), using (2.11) or (2.16) with $\gamma \equiv 0$, shows that

$$
\left\|u_{h}\right\|_{W_{p}^{1}\left(\Omega_{0}\right)} \leq K(p) \sup _{\substack{0 \neq v_{h} \in V_{h}^{0}\\}} \frac{\left\langle\nabla u_{h}, \nabla v_{h}\right\rangle}{\left\|v_{h}\right\|_{W_{q}^{1}\left(\Omega_{0}\right)}}
$$


for all $1 \leq p \leq \infty$, where

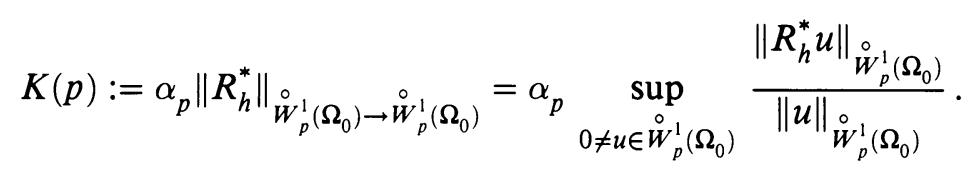

Here, we recall that

$$
\|u\|_{W_{p}^{1}\left(\Omega_{0}\right)}:=\left(\int_{\Omega_{0}}|\nabla u|^{p} d x\right)^{1 / p},
$$

and we note that the constant $\alpha_{p}$ from (2.16) was observed to be log-convex as a function of $1 / p$ by Meyers [17].

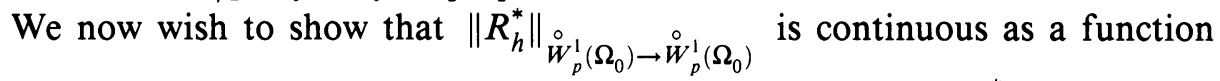
of $p$. To use Banach space interpolation theory, we view $R_{h}^{*}$ as inducing a mapping, $\mathscr{R}_{h}^{*}: \nabla u \longrightarrow \nabla u_{h}$, of $L^{p}(\Omega)^{2}$ to itself. More precisely, given $\mathbf{F} \in L^{p}(\Omega)^{2}$, let $u_{h}(\mathbf{F}) \in \stackrel{\circ}{V}_{h}^{0}$ solve

$$
\left\langle\nabla u_{h}, \nabla v_{h}\right\rangle=\left\langle\mathbf{F}, \nabla v_{h}\right\rangle \quad \forall v_{h} \in \stackrel{\circ}{V}_{h}^{0} .
$$

Then $\mathscr{R}_{h}^{*}(\mathbf{F}):=\nabla u_{h}$. Similarly, we can let $u \in \stackrel{\circ}{W}_{p}^{1}\left(\Omega_{0}\right)$ solve

$$
\langle\nabla u, \nabla v\rangle=\langle\mathbf{F}, \nabla v\rangle \quad \forall v \in \stackrel{\circ}{W}_{q}^{1}\left(\Omega_{0}\right)
$$

and we have from [16] that

$$
\|u\|_{\stackrel{\circ}{p}_{p}^{1}\left(\Omega_{0}\right)} \leq \alpha_{p}\|\mathbf{F}\|_{L^{p}(\Omega)^{2}}
$$

Therefore,

$$
\left\|\mathscr{R}_{h}^{*} \mathbf{F}\right\|_{L^{p}(\Omega)^{2}} \leq C(p)\|\mathbf{F}\|_{L^{p}(\Omega)^{2}},
$$

where $C(p) \leq C^{*} \alpha_{p}$ and $C(2)=1$.

Using operator interpolation, we conclude that

$$
\left\|\mathscr{R}_{h}^{*} \mathbf{F}\right\|_{\left[L^{2}(\Omega)^{2}, L^{P}(\Omega)^{2}\right]_{\theta}} \leq C(P)^{\theta}\|\mathbf{F}\|_{\left[L^{2}(\Omega)^{2}, L^{P}(\Omega)^{2}\right]_{\theta}} .
$$

Whether using the real (with appropriate second index) or complex interpolation method [6],

$$
c(P)^{-1}\|\mathbf{F}\|_{L^{2(1-\theta)+P \theta}(\Omega)^{2}} \leq\|\mathbf{F}\|_{\left[L^{2}(\Omega)^{2}, L^{P}(\Omega)^{2}\right]_{\theta}} \leq c(P)\|\mathbf{F}\|_{L^{2(1-\theta)+P \theta}(\Omega)^{2}}
$$

where $c(2)=1$ and $c(p)$ is a continuous function of $p$ near $p=2$. Therefore,

$$
\left\|R_{h}^{*}\right\|_{\stackrel{\circ}{W}_{p}^{1}\left(\Omega_{0}\right) \rightarrow \stackrel{\circ}{W}_{p}^{1}\left(\Omega_{0}\right)} \leq c(P)^{2} C(P)^{\theta}, \quad p=2(1-\theta)+P \theta
$$

Thus, for all $\varepsilon>0$ there exists $P>2$ such that $K(p) \leq 1+\varepsilon$ for $2<p<P$, where $\varepsilon$ and $P$ are independent of $h$. 
We now consider the general case via a perturbation argument. Define a bilinear form $\mathbb{B}: W_{p}^{1}\left(\Omega_{0}\right) \times W_{q}^{1}\left(\Omega_{0}\right) \rightarrow \mathbb{R}$ by

$$
\mathbb{B}(u, v):=\langle\nabla u, \nabla v\rangle-\frac{1}{M} a(u, v),
$$

where $M$ satisfies (2.15). It follows from (2.14)-(2.15) that

$$
\mathbb{B}(u, v)=\int_{\Omega_{0}} \sum_{i, j=1}^{2} B_{i j}(\xi, \eta) \partial_{i} u \partial_{j} v d \xi d \eta,
$$

where the eigenvalues of $B$ are in $[0,1-\Lambda / M]$ a.e. and $\Lambda$ satisfies (2.14). Note that $\Lambda / M=1 / M^{2} \leq 1 / 2$. Therefore, Hölder's inequality implies

$$
\left|\mathbb{B}\left(u_{h}, v_{h}\right)\right| \leq \kappa_{p}\left(1-\frac{\Lambda}{M}\right)\left\|u_{h}\right\|_{W_{p}^{1}\left(\Omega_{0}\right)}\left\|v_{h}\right\|_{W_{q}^{1}\left(\Omega_{0}\right)},
$$

where $\kappa_{p}$ is a smooth function of $p($ near $p=2)$ satisfying $\kappa_{2}=1$. Using the identity

$$
\langle\nabla u, \nabla v\rangle=\mathbb{B}(u, v)+\frac{1}{M} a(u, v),
$$

together with estimates (3.15) and (3.16), yields

$$
\left(\frac{1}{K(p)}-\kappa_{p}\left(1-\frac{\Lambda}{M}\right)\right)\left\|u_{h}\right\|_{W_{p}^{1}\left(\Omega_{0}\right)} \leq \frac{1}{M} \sup _{\substack{0 \neq v_{h} \in \dot{V}_{h}^{0}\\}} \frac{a\left(u_{h}, v_{h}\right)}{\left\|v_{h}\right\|_{W_{q}^{1}\left(\Omega_{0}\right)}} .
$$

In view of the continuity of $K$ and $\kappa$ and the fact that $K(2)=\kappa_{2}=1$, there exists $P>2$ such that for any $p \in[2, P], 1 / K(p)-\kappa_{p}(1-\Lambda / M)>0$. Choosing

$$
\alpha(p)=\frac{K(p)}{M\left[1-\kappa_{p}(1-\Lambda / M) K(p)\right]},
$$

we complete the proof using the equivalence of norms

$$
\|\cdot\|_{W_{p}^{1}\left(\Omega_{0}\right)} \simeq\|\cdot\|_{W_{p}^{1}\left(\Omega_{0}\right)} \text { and }\|\cdot\|_{W_{q}^{1}\left(\Omega_{0}\right)} \simeq\|\cdot\|_{W_{q}^{1}\left(\Omega_{0}\right)}
$$

for functions vanishing on the boundary.

Proposition 3.5. Suppose $\left\|\gamma_{h}\right\|_{W_{\infty}^{1}(0,1)}<1 / 2$. Then there are constants $\alpha<\infty$, $2<P$ and $h_{0}>0$ such that for all $0<h \leq h_{0}$ and for any $u_{h} \in \stackrel{\circ}{V}_{h}^{\gamma_{h}}$

$$
\left\|u_{h}\right\|_{W_{p}^{1}\left(\Omega_{\gamma_{h}}^{h}\right)} \leq \alpha \sup _{0 \neq v_{h} \in \dot{V}_{h}^{\text {h }}} \frac{a_{\gamma_{h}}\left(u_{h}, v_{h}\right)}{\left\|v_{h}\right\|_{W_{q}^{1}\left(\Omega_{\gamma_{h}}^{h}\right)}^{h}},
$$

whenever $2 \leq p \leq P$. 
Proof. Transform the bilinear form $a_{\gamma_{h}}$ to a bilinear form in $\Omega_{0}$, that is,

$$
\begin{aligned}
a_{\gamma_{h}}\left(u_{h}, v_{h}\right) & =\left.\left.\sum_{i=1}^{N_{h}} \int_{K} \nabla u_{h}\right|_{K} \cdot \nabla v_{h}\right|_{K} d x d y \\
& =\sum_{i=1}^{N_{h}} \int_{\widetilde{K}} \sum_{l, m=1}^{2} A_{l m}^{h}\left(\xi, \eta ; \gamma_{h}\right) \partial_{l} \tilde{u}_{h} \partial_{m} \tilde{v}_{h} d \xi d \eta \\
& =: \tilde{a}_{h}\left(\tilde{u}_{h}, \tilde{v}_{h} ; \gamma_{h}\right),
\end{aligned}
$$

where $\left.A^{h}\right|_{K}=\left(D_{K} D_{K}^{T}\right)^{-1}\left|\operatorname{det} D_{K}\right|$ (cf. Proposition 3.1). In view of Proposition 3.1 , we may apply Proposition 3.3 again. The proposition follows from the equivalence, (3.1), of the norms $\|v\|_{W_{q}^{1}\left(\Omega_{\gamma}^{h}\right)}$ and $\|\tilde{v}\|_{W_{q}^{1}\left(\Omega_{0}\right)}$.

Define

$$
V_{\varepsilon}^{h}=\left\{\left(\gamma_{h}, u_{h}\right) \in S_{h} \times V_{h}^{\gamma_{h}}:\left\|\gamma_{h}\right\|_{W_{\infty}^{1}(0,1)}<1 / 2,\left\|u_{h}\right\|_{W_{p}^{1}\left(\Omega_{\gamma_{h}}^{h}\right)}<\varepsilon\right\},
$$

and consider the transformation $T_{h}$ that associates with an element $\left(\gamma_{h}, u_{h}\right) \in$ $V_{\varepsilon}^{h}$ an element $\left(\gamma_{h}^{*}, u_{h}^{*}\right)$ which satisfies

$$
\begin{gathered}
b\left(\gamma_{h}^{*}, \chi_{h}\right)=a_{\gamma_{h}}\left(u_{h}, E_{\gamma_{h}}^{h} \chi_{h}\right) \quad \forall \chi_{h} \in S_{h}, \\
u_{h}^{*} \in g_{h}^{\gamma_{h}^{*}} \oplus \stackrel{\circ}{\gamma_{h}^{\gamma_{h}}} \text { such that } a_{\gamma_{h}^{*}}\left(u_{h}^{*}, v_{h}\right)=0 \quad \forall v_{h} \in \stackrel{\circ}{V}_{h}^{\gamma_{h}} .
\end{gathered}
$$

Proposition 3.6. Let $p$ be as in Theorem 2.1. There exist two strictly positive constants $\delta$ and $\varepsilon$ such that if

$$
\|g\|_{W_{p}^{1}\left(\Omega^{*}\right)}<\delta
$$

then $T_{h}$ is a mapping from $V_{\varepsilon}^{h} \rightarrow V_{\varepsilon}^{h}$.

Proof. Let $\left(\gamma_{h}, u_{h}\right) \in V_{\varepsilon}^{h}$. The variational problem, to find $\gamma_{h}^{*} \in S_{h}$ such that

$$
b\left(\gamma_{h}^{*}, \chi_{h}\right)=a_{\gamma_{h}}\left(u_{h}, E_{\gamma_{h}}^{h} \chi_{h}\right) \quad \forall \chi_{h} \in S_{h},
$$

admits a unique solution in $S_{h}$ because $b$ is a positive-definite, bilinear form in $S_{h} \times S_{h}$ and the functional

$$
\phi\left(\chi_{h}\right)=a_{\gamma_{h}}\left(u_{h}, E_{\gamma_{h}}^{h} \chi_{h}\right)
$$

is continuous from $S_{h}$ to $\mathbb{R}$. From inequalities (3.10) and (3.8) we have the following estimate on $\gamma_{h}^{*}$ :

$$
\left\|\gamma_{h}^{*}\right\|_{W_{\infty}^{1}(0,1)} \leq C \beta \mu\left(\left\|\gamma_{h}\right\|_{W_{\infty}^{1}(0,1)}\right)\left\|u_{h}\right\|_{W_{p}^{1}\left(\Omega_{\gamma_{h}}^{h}\right)} \leq C \varepsilon .
$$

Choose $\varepsilon$ such that $C \varepsilon<1 / 2$. Hence $\left\|\gamma_{h}^{*}\right\|_{W_{\infty}^{1}(0,1)}<1 / 2$.

Next we bound $u_{h}^{*}$. As $a_{\gamma_{h}^{*}}$ is a positive-definite, continuous bilinear form on $V_{h}\left(\Omega_{\gamma_{h}^{*}}\right)$, there exists a unique $w_{h} \in \stackrel{\circ}{V}_{h}\left(\Omega_{\gamma_{h}^{*}}\right)$ such that

$$
a_{\gamma_{h}^{*}}\left(w_{h}, v_{h}\right)=-a_{\gamma_{h}^{*}}\left(g_{h}^{\gamma_{h}^{*}}, v_{h}\right) \quad \forall v_{h} \in{\stackrel{\circ}{V_{h}^{*}}}_{\gamma_{h}^{*}} .
$$


In view of inequality (3.20) for $\gamma_{h}^{*}$, Proposition 3.5 can be used to bound $\left\|w_{h}\right\|_{W_{p}^{1}\left(\Omega_{\gamma_{h}^{*}}^{h}\right)}:$

$$
\left\|w_{h}\right\|_{W_{p}^{1}\left(\Omega_{\gamma_{h}^{*}}^{h}\right)} \leq C \alpha\left\|g_{h}^{\gamma_{h}^{*}}\right\|_{W_{p}^{1}\left(\Omega_{\gamma_{h}^{*}}^{h}\right)} .
$$

Let $u_{h}^{*}=w_{h}+g_{h}^{\gamma_{h}^{*}} ;$ then

$$
\left\|u_{h}^{*}\right\|_{W_{p}^{1}\left(\Omega_{\gamma_{h}^{*}}^{h}\right)} \leq(1+C \alpha)\left\|g_{h}^{\gamma_{h}^{*}}\right\|_{W_{p}^{1}\left(\Omega_{\gamma_{h}^{*}}^{h}\right)} .
$$

From inequalities (3.6) and (3.20) and the assumptions that $\left(\gamma_{h}, u_{h}\right) \in V_{\varepsilon}^{h}$ and $\|g\|_{W_{p}^{1}\left(\Omega^{*}\right)}<\delta$, we find that

$$
\left\|u_{h}^{*}\right\|_{W_{p}^{1}\left(\Omega_{\gamma_{h}^{*}}\right)} \leq C \delta .
$$

Finally, choose $\delta$ such that $C \delta<\varepsilon$. Hence $\left(\gamma_{h}^{*}, u_{h}^{*}\right) \in V_{\varepsilon}^{h}$.

Theorem 3.1. If the norm of $g$ is small enough, $T_{h}$ is a contraction from $\widetilde{V}_{\varepsilon}^{h} \rightarrow$ $\widetilde{V}_{\varepsilon}^{h}$ for the norm $\||\cdot|\|$.

The definition of $\widetilde{V}_{\varepsilon}^{h}$ and the proof of this result are similar to the continuous case.

Corollary 3.1. If $\|g\|_{W_{p}^{1}\left(\Omega^{*}\right)}$ is sufficiently small, problem (3.9) admits a unique solution in $V_{\varepsilon}^{h}$ which can be calculated by a fixed point iteration,

$$
\left(\gamma_{h}^{i+1}, u_{h}^{i+1}\right)=T\left(\gamma_{h}^{i}, u_{h}^{i}\right)
$$

whenever $\left(\gamma_{h}^{0}, u_{h}^{0}\right) \in V_{\varepsilon}^{h}$.

As initial data $\left(\gamma_{h}^{0}, u_{h}^{0}\right)$, we can choose $\gamma_{h}^{0} \equiv 0$ in $[0,1]$ and $u_{h}^{0}$ as the Ritz projection, $R_{h}^{*} u^{0}$, of the solution, $u^{0}$, of problem (2.22). Clearly, for $h$ small enough, $\left(u_{h}^{0}, \gamma_{h}^{0}\right) \in V_{\varepsilon}^{h}$.

\section{Convergence results.}

Theorem 3.2. If the small-norm solution $(\gamma, u) \in V_{\varepsilon}$ of problem (2.9) for $p>2$ satisfies $\gamma \in W_{\infty}^{2}(0,1)$ and $\varepsilon$ is sufficiently small, there is a constant $C<\infty$ and a strictly positive $h_{0}$ such that for all $0<h \leq h_{0}$

$$
\begin{aligned}
& \left\|\gamma-\gamma_{h}\right\|_{W_{\infty}^{1}(0,1)}+\left\|\hat{u}-\tilde{u}_{h}\right\|_{W_{p}^{1}\left(\Omega_{0}\right)} \\
& \quad \leq C\left(h\|\gamma\|_{W_{\infty}^{2}(0,1)}+\inf _{\tilde{v} \in \tilde{g}_{h}^{\prime} \oplus \dot{V}_{h}^{0}}\|\hat{u}-\tilde{v}\|_{W_{p}^{1}\left(\Omega_{0}\right)}\right),
\end{aligned}
$$

where $\left(u_{h}, \gamma_{h}\right)$ is the small-norm solution of the discrete problem (3.9). 
Proof. Let $(\gamma, u) \in V_{\varepsilon_{1}}$ be the solution of problem (2.9), and let $\left(\gamma_{h}, u_{h}\right) \in V_{\varepsilon_{2}}^{h}$ be the solution of problem (3.9). Since $E \chi_{h}-J_{h} E \chi_{h} \in \stackrel{\circ}{W}_{q}^{1}\left(\Omega_{0}\right)$, we have

$$
\begin{aligned}
b(\gamma- & \left.\gamma_{h}, \chi_{h}\right)=a_{\gamma}\left(u, E_{\gamma} \chi_{h}\right)-a_{\gamma_{h}}\left(u_{h}, E_{\gamma_{h}}^{h} h \chi_{h}\right) \\
= & \hat{a}\left(\hat{u}, E \chi_{h} ; \gamma\right)-\tilde{a}_{h}\left(\tilde{u}_{h}, J_{h} E \chi_{h} ; \gamma_{h}\right) \\
= & \hat{a}\left(\hat{u}, J_{h} E \chi_{h} ; \gamma\right)-\tilde{a}_{h}\left(\tilde{u}_{h}, J_{h} E \chi_{h} ; \gamma_{h}\right) \\
= & \hat{a}\left(\hat{u}, J_{h} E \chi_{h} ; \gamma\right)-\tilde{a}_{h}\left(\hat{u}, J_{h} E \chi_{h} ; \gamma_{h}\right) \\
& +\tilde{a}_{h}\left(\hat{u}-\tilde{u}_{h}, J_{h} E \chi_{h} ; \gamma_{h}\right) \\
= & \int_{\Omega_{0}} \sum_{i, j=1}^{2}\left(A_{i j}(\xi, \eta ; \gamma)-A_{i j}^{h}\left(\xi, \eta ; \gamma_{h}\right)\right) \partial_{i} \hat{u} \partial_{j}\left(J_{h} E \chi_{h}\right) d \xi d \eta \\
& +\tilde{a}_{h}\left(\hat{u}-\tilde{u}_{h}, J_{h} E \chi_{h} ; \gamma_{h}\right) \\
= & \int_{\Omega_{0}} \sum_{i, j=1}^{2}\left(A_{i j}(\xi, \eta ; \gamma)-A_{i j}^{h}(\xi, \eta ; \gamma)\right) \partial_{i} \hat{u} \partial_{j}\left(J_{h} E \chi_{h}\right) d \xi d \eta \\
& +\int_{\Omega_{0}} \sum_{i, j=1}^{2}\left(A_{i j}^{h}(\xi, \eta ; \gamma)-A_{i j}^{h}\left(\xi, \eta ; \gamma_{h}\right)\right) \partial_{i} \hat{u} \partial_{j}\left(J_{h} E \chi_{h}\right) d \xi d \eta \\
& +\tilde{a}_{h}\left(\hat{u}-\tilde{u}_{h}, J_{h} E \chi_{h} ; \gamma_{h}\right) .
\end{aligned}
$$

The representations for $A$ and $A^{h}$ (see (2.13) and (3.18), respectively) imply that

$$
\begin{aligned}
\left|A_{i j}(\xi, \eta ; \gamma)-A_{i j}^{h}(\xi, \eta ; \gamma)\right| & \leq C h\|\gamma\|_{W_{\infty}^{2}(0,1)}, \\
\left|A_{i j}^{h}(\xi, \eta ; \gamma)-A_{i j}^{h}\left(\xi, \eta ; \gamma_{h}\right)\right| & \leq C\left\|\gamma-\gamma_{h}\right\|_{W_{\infty}^{1}(0,1)} .
\end{aligned}
$$

Combining the estimates (3.23) with the identity (3.22) yields

$$
\begin{array}{rl}
\left|b\left(\gamma-\gamma_{h}, \chi_{h}\right)\right| \leq & C\left(h\|\gamma\|_{W_{\infty}^{2}(0,1)}+\left\|\gamma-\gamma_{h}\right\|_{W_{\infty}^{1}(0,1)}\right)\|\hat{u}\|_{W_{p}^{1}\left(\Omega_{0}\right)}\left\|J_{h} E \chi_{h}\right\|_{W_{q}^{1}\left(\Omega_{0}\right)} \\
& +\left|a_{h}\left(\hat{u}-\tilde{u}_{h}, J_{h} E \chi_{h} ; \gamma_{h}\right)\right| \\
\leq & C\left(\left(h\|\gamma\|_{W_{\infty}^{2}(0,1)}+\left\|\gamma-\gamma_{h}\right\|_{W_{\infty}^{1}(0,1)}\right)\|\hat{u}\|_{W_{p}^{1}\left(\Omega_{0}\right)}\right. \\
& \left.+\left\|\hat{u}-\tilde{u}_{h}\right\|_{W_{p}^{1}\left(\Omega_{0}\right)}\left\|\gamma_{h}\right\|_{W_{\infty}^{1}(0,1)}\right)\left\|J_{h} E \chi_{h}\right\|_{W_{q}^{1}\left(\Omega_{0}\right)} \\
\leq C & C\left(\left(h\|\gamma\|_{W_{\infty}^{2}(0,1)}+\left\|\gamma-\gamma_{h}\right\|_{W_{\infty}^{1}(0,1)}\right)\|\hat{u}\|_{W_{p}^{1}\left(\Omega_{0}\right)}\right. \\
& \left.+\left\|\hat{u}-\tilde{u}_{h}\right\|_{W_{p}^{1}\left(\Omega_{0}\right)}\left\|\gamma_{h}\right\|_{W_{\infty}^{1}(0,1)}\right)\left\|\chi_{h}\right\|_{W_{1}^{1}(0,1)} .
\end{array}
$$

Let $\psi \in S_{h}$. Since

$$
b\left(\psi-\gamma_{h}, \chi_{h}\right)=b\left(\gamma-\gamma_{h}, \chi_{h}\right)-b\left(\gamma-\psi, \chi_{h}\right),
$$

Proposition 3.2 and Hölder's inequality imply that

$$
\begin{aligned}
\left\|\psi-\gamma_{h}\right\|_{W_{\infty}^{1}(0,1)} \leq C\left(\left(h\|\gamma\|_{W_{\infty}^{2}(0,1)}+\left\|\gamma-\gamma_{h}\right\|_{W_{\infty}^{1}(0,1)}\right)\|\hat{u}\|_{W_{p}^{1}\left(\Omega_{0}\right)}\right. \\
\left.+\left\|\hat{u}-\tilde{u}_{h}\right\|_{W_{p}^{1}\left(\Omega_{0}\right)}\left\|\gamma_{h}\right\|_{W_{\infty}^{1}(0,1)}+\|\gamma-\psi\|_{W_{\infty}^{1}(0,1)}\right) .
\end{aligned}
$$


Subtracting and using the triangle inequality, we find

$$
\begin{gathered}
\left\|\gamma-\gamma_{h}\right\|_{W_{\infty}^{1}(0,1)}\left(1-C\|\hat{u}\|_{W_{p}^{1}\left(\Omega_{0}\right)}\right) \leq C\left(h\|\gamma\|_{W_{\infty}^{2}(0,1)}\|\hat{u}\|_{W_{p}^{1}\left(\Omega_{0}\right)}\right. \\
\left.+\left\|\hat{u}-\tilde{u}_{h}\right\|_{W_{p}^{1}\left(\Omega_{0}\right)}\left\|\gamma_{h}\right\|_{W_{\infty}^{1}(0,1)}+\|\gamma-\psi\|_{W_{\infty}^{1}(0,1)}\right) .
\end{gathered}
$$

Choosing $\varepsilon$ appropriately, we find

$$
\left\|\gamma-\gamma_{h}\right\|_{W_{\infty}^{1}(0,1)} \leq C\left(h\|\gamma\|_{W_{\infty}^{2}(0,1)}+\left\|\hat{u}-\tilde{u}_{h}\right\|_{W_{p}^{1}\left(\Omega_{0}\right)}+\|\gamma-\psi\|_{W_{\infty}^{1}(0,1)}\right) .
$$

Choosing $\psi=I_{h} \gamma$ and using (3.2), we have

$$
\left\|\gamma-\gamma_{h}\right\|_{W_{\infty}^{1}(0,1)} \leq C\left(h\|\gamma\|_{W_{\infty}^{2}(0,1)}+\left\|\hat{u}-\tilde{u}_{h}\right\|_{W_{p}^{1}\left(\Omega_{0}\right)}\right) .
$$

Next we bound $\left\|\hat{u}-\tilde{u}_{h}\right\|_{W_{p}^{1}\left(\Omega_{0}\right)}$. Using Proposition 3.5, or equivalently Proposition 3.3, we find for arbitrary $\tilde{v}_{h} \in g_{h}^{\gamma_{h}} \oplus \stackrel{\circ}{V}_{h}^{0}$ that

$$
\begin{aligned}
& \left\|\tilde{v}_{h}-\tilde{u}_{h}\right\|_{W_{p}^{1}\left(\Omega_{0}\right)} \leq C \sup _{\substack{0 \neq w_{h} \in V_{h}^{0}}} \frac{\tilde{a}_{h}\left(\tilde{v}_{h}-\tilde{u}_{h}, w_{h} ; \gamma_{h}\right)}{\left\|w_{h}\right\|_{W_{q}^{1}\left(\Omega_{0}\right)}}
\end{aligned}
$$

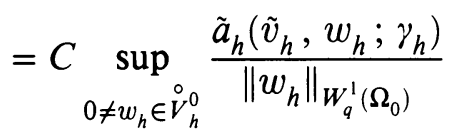

$$
\begin{aligned}
& =C \sup _{0 \neq w_{h} \in V_{h}^{0}} \frac{\tilde{a}_{h}\left(\tilde{v}_{h}-\hat{u}, w_{h} ; \gamma_{h}\right)+\tilde{a}_{h}\left(\hat{u}, w_{h} ; \gamma_{h}\right)}{\left\|w_{h}\right\|_{W_{q}^{\prime}\left(\Omega_{0}\right)}} \\
& =C \sup _{0 \neq w_{h} \in V_{h}^{0}} \frac{\tilde{a}_{h}\left(\tilde{v}_{h}-\hat{u}, w_{h} ; \gamma_{h}\right)+\tilde{a}_{h}\left(\hat{u}, w_{h} ; \gamma_{h}\right)-\hat{a}\left(\hat{u}, w_{h} ; \gamma\right)}{\left\|w_{h}\right\|_{W_{q}^{1}\left(\Omega_{0}\right)}} .
\end{aligned}
$$

As above, we have

$$
\begin{aligned}
\mid \tilde{a}_{h}(\hat{u}, & \left.w_{h} ; \gamma_{h}\right)-\hat{a}\left(\hat{u}, w_{h} ; \gamma\right) \mid \\
\quad & \left|\int_{\Omega_{0}} \sum_{i, j=1}^{2}\left(A_{i j}^{h}\left(\xi, \eta ; \gamma_{h}\right)-A_{i j}(\xi, \eta ; \gamma)\right) \partial_{i} \hat{u} \partial_{j} w_{h} d \xi d \eta\right| \\
& \leq C\left(h\|\gamma\|_{W_{\infty}^{2}(0,1)}+\left\|\gamma-\gamma_{h}\right\|_{W_{\infty}^{1}(0,1)}\right)\|\hat{u}\|_{W_{p}^{1}\left(\Omega_{0}\right)}\left\|w_{h}\right\|_{W_{q}^{1}\left(\Omega_{0}\right)} .
\end{aligned}
$$

By Hölder's inequality, we thus find

$$
\begin{aligned}
\left\|\tilde{v}_{h}-\tilde{u}_{h}\right\|_{W_{p}^{1}\left(\Omega_{0}\right)} \leq & C\left\|\tilde{v}_{h}-\hat{u}\right\|_{W_{p}^{1}\left(\Omega_{0}\right)} \\
& +C\left(h\|\gamma\|_{W_{\infty}^{2}(0,1)}+\left\|\gamma-\gamma_{h}\right\|_{W_{\infty}^{1}(0,1)}\right)\|\hat{u}\|_{W_{p}^{1}\left(\Omega_{0}\right)} .
\end{aligned}
$$

From the triangle inequality, we have

$$
\begin{aligned}
\left\|\hat{u}-\tilde{u}_{h}\right\|_{W_{p}^{1}\left(\Omega_{0}\right)} \leq & (1+C)\left\|\tilde{v}_{h}-\hat{u}\right\|_{W_{p}^{1}\left(\Omega_{0}\right)} \\
& +C\left(h\|\gamma\|_{W_{\infty}^{2}(0,1)}+\left\|\gamma-\gamma_{h}\right\|_{W_{\infty}^{1}(0,1)}\right)\|\hat{u}\|_{W_{p}^{1}\left(\Omega_{0}\right)} .
\end{aligned}
$$

Applying the previous estimate for $\gamma-\gamma_{h}$ and choosing $\varepsilon$ sufficiently small, we find

$$
\left\|\hat{u}-\tilde{u}_{h}\right\|_{W_{p}^{1}\left(\Omega_{0}\right)} \leq C\left(h\|\gamma\|_{W_{\infty}^{2}(0,1)}+\left\|\tilde{v}_{h}-\hat{u}\right\|_{W_{p}^{1}\left(\Omega_{0}\right)}\right) .
$$


Combining this with the previous estimate for $\gamma-\gamma_{h}$ completes the proof.

Corollary 3.2. If the small-norm solution $(\gamma, u) \in V_{\varepsilon}$ of problem (2.9) satisfies $(\gamma, u) \in W_{\infty}^{2}(0,1) \times W_{p}^{2}\left(\Omega_{\gamma}\right)$ for $p>2$ and $\varepsilon$ is sufficiently small, there is a constant $C<\infty$ and a strictly positive $h_{0}$ such that for all $0<h \leq h_{0}$

$$
\left\|\hat{u}-\tilde{u}_{h}\right\|_{W_{p}^{1}\left(\Omega_{0}\right)}+\left\|\gamma-\gamma_{h}\right\|_{W_{\infty}^{1}(0,1)} \leq C h\left(\|\gamma\|_{W_{\infty}^{2}(0,1)}+\|\hat{u}\|_{W_{p}^{2}\left(\Omega_{0}\right)}\right) .
$$

Proof. We have $I_{h}^{0} \hat{u} \in \tilde{g}_{h}^{\gamma} \oplus \stackrel{\circ}{V}_{h}^{0}$, so the result follows from (3.5).

\section{CONCLUSIONS AND EXTENSIONS}

We have demonstrated optimal-order convergence of a finite element approximation for a model free-boundary problem using piecewise linear approximation for both the field variable, $u$, and the free-surface representation function, $\gamma$. It would therefore appear appropriate to choose the order of approximation to be the same in this case.

We can extend this work to models involving the curvature of the free surface, instead of the expression given in the third line of (1.1). This requires only the introduction of a nonlinearity in the bilinear form, $b$. The definition of $b$ depends on the chosen parameterization of the free surface, but one particular case would be

$$
b(\gamma, \chi):=s \int_{0}^{1} \frac{\gamma^{\prime}(x) \chi^{\prime}(x)}{\left[1+\gamma^{\prime}(x)^{2}\right]^{1 / 2}} d x .
$$

The model in differential form corresponds to the following equation for the free surface:

$$
-s\left(\frac{\gamma^{\prime}(x)}{\left[1+\gamma^{\prime}(x)^{2}\right]^{1 / 2}}\right)^{\prime}=\frac{\partial u(x, 1+\gamma(x))}{\partial \vec{n}}\left[1+\gamma^{\prime}(x)^{2}\right]^{1 / 2} \quad \forall x \in[0,1] .
$$

With this replacement, the results in the previous sections would remain the same. If the equation for the free surface were

$$
-s\left(\frac{\gamma^{\prime}(x)}{\left[1+\gamma^{\prime}(x)^{2}\right]^{1 / 2}}\right)^{\prime}=\frac{\partial u(x, 1+\gamma(x))}{\partial \vec{n}} \quad \forall x \in[0,1],
$$

the extension operator $E_{\gamma}$ would be more complicated, namely, $E_{\gamma} \chi$ would be an extension of $\chi /\left[1+\gamma^{\prime}(x)^{2}\right]^{1 / 2}$ instead of just $\chi$, but the results would be similar. Other models (cf. [16]) could be treated similarly.

Models for which the derivative of $\gamma$ are specified at the endpoints of the free surface are more complicated. Although Lemma 2.0 implies that the solution to both the continuous and discrete models will exist in this case, it is not clear that convergence will be optimal-order. The corresponding difficulty arises at the first step of the proof of Theorem 3.2 in that we can no longer assure that $E \chi_{h}-J_{h} E \chi_{h} \in \stackrel{\circ}{W}_{q}^{1}\left(\Omega_{0}\right)$. The discrete extension as defined here would necessarily be nonzero on one edge on the side boundary (where $E \chi_{h}$ vanishes), 
assuming the test function, $\chi_{h}$, were not to vanish there. It may be possible to avoid this difficulty in some way, either by a different analysis or by a different choice of discrete extension. However, at the moment this is an open problem.

We wish to develop a theory for more general models of viscous, incompressible flow. Formally, the techniques used to convert the free-boundary problem (1.1) in differential form can be used to develop a variational form for the full Navier-Stokes equations. However, a key result needed to prove the stability of the Galerkin method for the problem (1.1) is the bound for the maximum norm of the gradient for the Galerkin method applied to a scalar elliptic problem [21]. Such bounds are not yet known for the Stokes equations (cf. [12]), so such estimates need to be derived or an alternative technique needs to be developed. Another extension we wish to make is by proving local (quadratic) convergence of the Newton iteration (cf. [24]) and other efficient iterative procedures for solving the discrete system corresponding to models such as these. Finally, variational formulations need to be developed for problems involving boundary data that are allowed to be nonzero at the point of attachment of the free surface to the boundary on which Dirichlet conditions are imposed. If care is not taken, such boundary conditions can be incompatible, leading to singular solutions.

\section{ACKNOWLEDGMENTS}

The authors wish to thank both Professors W. G. Pritchard and L. E. Scriven for having introduced them to free-surface problems and for valuable comments concerning this work.

\section{BIBLIOGRAPHY}

1. G. Albinus, $L^{p}$ coercivity in plane domains with piecewise smooth boundary, Math. Nachr. 118 (1984), 317-336.

2. G. Allain, Small-time existence for the Navier-Stokes equations with a free surface, Appl. Math. Optim. 16 (1987), 37-50.

3. D. N. Arnold, L. R. Scott, and M. Vogelius, Regular inversion of the divergence operator with Dirichlet boundary conditions on a polygon, Ann. Scuola Norm. Sup. Pisa Cl. Sci. (4) 15 (1988), 169-192.

4. J. T. Beale, Large-time regularity of viscous surface waves, Arch. Rational Mech. Anal. 84 (1984), 307-352.

5. J. Bemelmans, Nonstationary viscous fluid flow generated by surface tension I: Existence of classical solution, Univ. Bonn SFB Preprint no. 509, 1982.

6. Joran Bergh and Jorgen Löfstrom, Interpolation spaces, an introduction, Springer-Verlag, Berlin, 1976.

7. H. Brezis, Analyse fonctionnelle, Masson Editeur, 1983.

8. P. G. Ciarlet, The finite element method for elliptic problems, North-Holland, Amsterdam, 1978.

9. P. Clement, Approximation by finite element functions using local regularization, R.A.I.R.O. Anal. Numér. Ser. Rouge 9 (1975), 77-84.

10. C. Cuvelier, On the numerical solution of a capillary free boundary problem governed by the Navier-Stokes equations, Lecture Notes in Phys., vol. 141, Springer-Verlag, Berlin and New York, 1980, pp. 132-137. 
11. J. Douglas, Jr., T. Dupont, and L. Wahlbin, Optimal $L_{\infty}$ error estimates for Galerkin approximations to solutions of two-point boundary value problems, Math. Comp. 29 (1975), 475-483.

12. R. Durán, R. H. Nochetto, and J. Wang, Sharp maximum norm error estimates for finite element approximations of the Stokes problem in 2-D, Math. Comp. 51 (1988), 491-506.

13. P. Grisvard, Elliptic problems in nonsmooth domains, Pitman Advanced Publishing Program, Boston, 1985.

14. M. Jean, Free surface of the steady flow of a Newtonian fluid in a finite channel, Arch. Rational Mech. Anal. 74 (1980), 197-217.

15. M. Jean and W.G. Pritchard, The flow of fluids from nozzles at small Reynolds numbers, Proc. Roy. Soc. London Ser. A 370 (1980), 61-72.

16. J. B. Keller and M. J. Miksis, Surface tension driven flows, SIAM J. Appl. Math. 43 (1983), 268-277.

17. N. G. Meyers, $A n L^{p}$-estimate for the gradient of solutions of second order elliptic divergence equations, Ann. Scuola Norm. Sup. Pisa Ser. III 17 (1963), 189-206.

18. J. A. Nitsche, Free boundary problems for Stokes' flows and finite element methods, Equadiff 6, Lecture Notes in Math., vol. 1192, Springer-Verlag, Berlin, 1986, pp. 327-332.

19. W. G. Pritchard, Instability and chaotic behaviour in a free-surface flow, J. Fluid Mech. 165 (1986), 1-60.

20. V. V. Pukhnachov [Pukhnachëv], Hydrodynamic free boundary problems, Nonlinear Partial Differential Equations and their Applications, Collège de France Seminar Volume III, Pitman, Boston, 1982, pp. 301-308.

21. R. Rannacher and R. Scott, Some optimal error estimates for piecewise linear finite element approximations, Math. Comp. 38 (1982), 437-445.

22. P. A. Raviart and V. Girault, Finite element method for Navier-Stokes equations. Theory and algorithms, Springer-Verlag, Berlin and New York, 1986.

23. G. Ryskin and L. G. Leal, Numerical solution of free-boundary problems in fluid mechanics, Parts 1-3, J. Fluid Mech. 148 (1984), 1-43.

24. H. Saito and L. E. Scriven, Study of coating flow by the finite element method, J. Comput. Phys. 42 (1981), 53-76.

25. R. Scott and S. Zhang, Finite element interpolation of nonsmooth functions satisfying boundary conditions, Math. Comp. 54 (1990), 483-493.

26. C. G. Simader, On Dirichlet's boundary value problem, Lecture Notes in Math., vol. 268, Springer-Verlag, Berlin, 1972.

27. V. A. Solonnikov, On the Stokes equations in domains with non-smooth boundaries and on viscous incompressible flow with a free surface, Nonlinear Partial Differential Equations and their Applications, Collège de France Seminar Volume III, Pitman, Boston, 1982, pp. 340-423.

28. FIDAP Theoretical Manual, Revision 4.0, Fluid Dynamics International, Evanston, IL, 1987.

Departamento de Matematicas, Universidad Autonoma Metropolitana, Appdo. Postal 55-534, Mexico, 09340, D. F. Mexico

Department of Mathematics, University of Houston, Houston, Texas 77204.

E-mail address: scott@uh.edu 